\title{
From Jöbsis to the present day: a review of clinical near-infrared spectroscopy measurements of cerebral cytochrome-c-oxidase
}

Gemma Bale

Clare E. Elwell

Ilias Tachtsidis 


\title{
From Jöbsis to the present day: a review of clinical near-infrared spectroscopy measurements of cerebral cytochrome-c-oxidase
}

\author{
Gemma Bale, ${ }^{*}$ Clare E. Elwell, and Ilias Tachtsidis \\ University College London, Engineering Faculty, Department of Medical Physics and Biomedical Engineering, Malet Place Engineering Building, \\ Malet Place, London WC1E 6BT, United Kingdom
}

\begin{abstract}
Near-infrared spectroscopy (NIRS) measurements of cytochrome-c-oxidase (CCO) have the potential to yield crucial information about cerebral metabolism at the patient bedside. Developments in instrumentation and the analytical methods used to resolve changes in CCO have led to many clinical applications of the measurement since its first demonstration in 1977 by Jöbsis. There is a substantial literature of work on measures of $\mathrm{CCO}$ in animal and in vitro studies; however, this review focuses on translational studies. Almost 40 years from the advent of the first measurement of CCO using NIRS, this signal continues to hold significant interest in our understanding of the human brain in health and disease. We discuss methodologies for obtaining NIRS measurements of CCO in the clinic and review studies in neonates and adults. $\odot$ The Authors. Published by SPIE under a Creative Commons Attribution 3.0 Unported License. Distribution or reproduction of this work in whole or in part requires full attribution of the original publication, including its DOI. [DOI: 10.1117/1.JBO.21.9.091307]
\end{abstract}

Keywords: infrared spectroscopy; biomedical optics; medical imaging.

Paper 160032SSVR received Jan. 16, 2016; accepted for publication Apr. 1, 2016; published online May 11, 2016; corrected May 26, 2016.

\section{Introduction}

A patient's health is in great danger when there is a prolonged lack of oxygen delivery to meet the metabolic demand of the tissue; even brief periods of hypoxia can cause permanent damage to brain tissue. This is a huge problem in patients with acute brain injury, for example, where derangements of cerebral oxygen delivery and utilization often occur, rendering the brain susceptible to secondary, or additional, injury processes. Secondary injury is also common in neonatal brain injury after perinatal hypoxia-ischemia (HI), affecting neurodevelopmental outcome. A number of hemoglobin-based oxygenation monitors are available; however, clinicians currently cannot monitor the biochemical status of the injured brain continuously and noninvasively at the bedside. There is therefore an urgent need for real-time, in vivo measurements of brain tissue oxygenation and biochemistry in the clinic; a need for a bedside sensor that can improve diagnostic information and indicate when to alter and/or redirect therapy to improve clinical outcome.

Since 1977, when Franz Jöbsis reported a new optical method in a seminal Science article, ${ }^{1}$ near-infrared spectroscopy (NIRS) has been seen as the technique, which could deliver a solution to this clinical need. NIRS has become an established research and clinical tool for measuring changes in cerebral oxygenation, in particular, changes in oxygenated and deoxygenated hemoglobin $\left(\mathrm{HbO}_{2}\right.$ and $\left.\mathrm{HHb}\right)$ concentration. This groundbreaking paper has been cited close to 3000 times. However, Jöbsis's intention was to develop an optical technique to measure in vivo changes in cytochrome-c-oxidase (CCO), an enzyme in the mitochondria, and hence monitor tissue metabolism (see his candid account of his discovery ${ }^{2}$ ). Jöbsis discovered that near-infrared (NIR) light

*Address all correspondence to: Gemma Bale, E-mail: gemma.bale.11@ucl.ac .uk penetrates deep into living tissue and this optical window could be used to monitor changes in the concentrations of absorbing compounds inside the tissue. The tissue light attenuation measurements are related to changes in the concentrations of $\mathrm{HbO}_{2}$ and $\mathrm{HHb}$, and the redox state of CCO. The legacy of Jöbsis's work lies in the measurement of changes in hemoglobin oxygenation with NIRS, as it is much simpler to measure the hemoglobin chromophores, which are present in high concentrations compared to CCO; but his goal of an in vivo monitor of tissue metabolism is as important today as it was almost four decades ago.

There has been a significant amount of work on measuring hemoglobin concentration changes with NIRS since 1977 (see the recent reviews by Wolf et $\mathrm{al}^{3}{ }^{3}$ and Scholkmann et al. ${ }^{4}$ ). A typical commercially available NIRS system is a noninvasive, inexpensive, portable, bedside monitor that can measure, at multiple sites, the changes in $\mathrm{HbO}_{2}$ and $\mathrm{HHb}$ via the modified BeerLambert law (described in Ref. 4). From those, it is possible to derive estimations of changes in oxygen delivery (via hemoglobin difference, $\mathrm{HbD}=\mathrm{HbO}_{2}-\mathrm{HHb}$ ) and changes in total blood volume (total hemoglobin, $\mathrm{HbT}=\mathrm{HbO}_{2}+\mathrm{HHb}$ ). In addition, from technical developments in the late 1990s, we now have clinical NIRS cerebral oximeters that measure absolute tissue saturation [known as cerebral oxygen saturation $\left(\mathrm{ScO}_{2}\right)$, tissue oxygen saturation $\left(\mathrm{StO}_{2}\right)$, or tissue oxygenation index (TOI)], which is the ratio of absolute $\mathrm{HbO}_{2}$ and absolute $\mathrm{HbT}$, using techniques, such as spatially resolved spectroscopy. ${ }^{5,6}$ However, these measurements do not monitor metabolism at a cellular level unlike a measurement of the redox state of $\mathrm{CCO}$, which has the potential to yield an in vivo indication of cellular energy metabolism. Simultaneous measurement of the CCO and hemoglobin signals can therefore provide complimentary information on hemodynamics, oxygenation, and metabolism. Monitoring of $\mathrm{CCO}$ with NIRS has been shown to be consistent with other measures of metabolism; including those from more invasive techniques, 
such as microdialysis in humans, ${ }^{7}$ or more expensive and less portable techniques, such as magnetic resonance spectroscopy (MRS) in animal studies. ${ }^{8-11}$ Furthermore, the concentration of $\mathrm{CCO}$ is much higher in the brain than in extracerebral tissues, so it is a more brain-specific signal that is less prone to scalp and skull contamination than the hemoglobin signals. ${ }^{12,13}$ This has potentially huge importance for functional NIRS (fNIRS) studies in which the hemoglobin signals suffer from contamination from the extracerebral tissues. ${ }^{14}$

Preclinical and in vitro studies have contributed enormously to our understanding of the optical issues surrounding the NIRS measurement of $\mathrm{CCO}$, its role as marker of cellular metabolism, and its potential as a clinically relevant measure. This was a focus for several research groups in the 1980s and 1990s. ${ }^{15-21}$ More recently, a study in a piglet model of $\mathrm{HI}$ brain injury has demonstrated the specificity of the $\mathrm{CCO}$ signal as a marker of tissue metabolism. ${ }^{10}$ In this study, the percentage recovery of $\mathrm{CCO}$ after $\mathrm{HI}$ to baseline levels was highly correlated with percentage recovery of phosphorus $\left({ }^{31} \mathrm{P}\right)$ MRS measures of cellular metabolism (see Fig. 1). Furthermore, this study demonstrates the potential for CCO measures to identify outcome after injury. However, in this review, we focus on the translational clinical and human cerebral studies that have been ongoing since $1985^{22}$ and we aim to identify and summarize this work from then until the present day.

Figure 2 shows how the number of publications on and the populations studied by NIRS-measured CCO has varied over the years. Between 1977 and 1997, there was a gradual increase in the number of papers published, varying in subject and research group [Duke University, University College London (UCL), Hokkaido University, Sapporo, and Keele University were home to the main groups involved]. After 1997, the interest in the CCO measurement gradually declined due to both controversies over the optical instrumentation, methods and algorithms used to resolve it, ${ }^{23}$ and a shift of clinical interest due

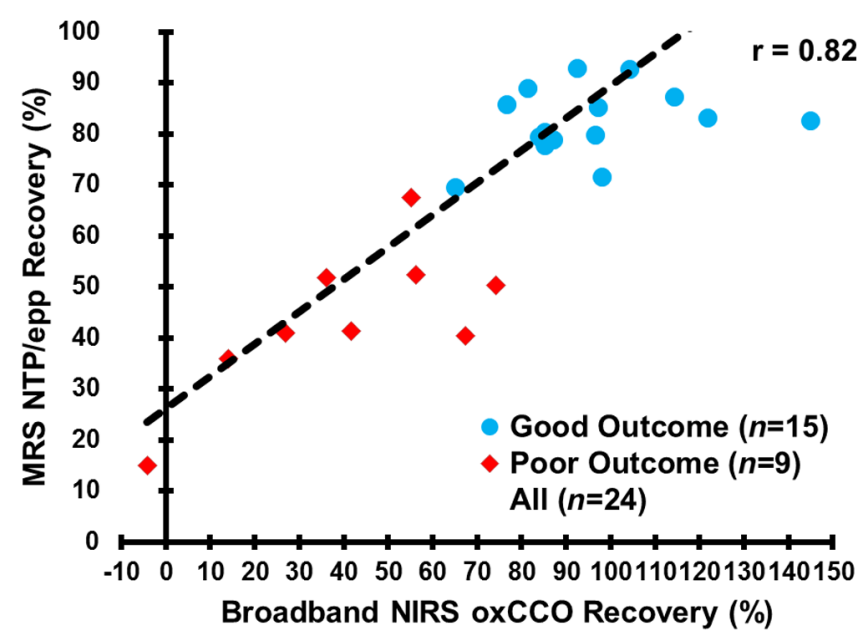

Fig. 1 Measured changes in oxCCO (see Sec. 2 for a full explanation of this term) following hypoxia-ischemia ( $\mathrm{HI})$ in a piglet model. Each data point represents the recovery fraction of the broadband NIRS oxCCO signal and the ${ }^{31} \mathrm{P}$ MRS NTP/epp (a surrogate marker of ATP). The broadband oxCCO measurements show a strong association (Pearson correlation, $r=0.82$ ) with the ${ }^{31} \mathrm{P}$ MRS metabolic measurements and provide a good indication of outcome (good outcome refers to piglets that survived for more than $24 \mathrm{~h}$ following $\mathrm{HI}$ ). The figure has been produced from data presented by Bainbridge et al. ${ }^{10}$ to the emergence of the absolute tissue saturation measurement with NIRS. ${ }^{5}$ In the 2000 s, efforts were made to optimize NIRS instrumentation and methods specifically for the measurement of $\mathrm{CCO}^{24,25}$ and studies were performed in healthy volunteers and patients to characterize the behavior of the CCO signal as a marker of cerebral cellular oxygen metabolism. ${ }^{7,13}$ A resurgence in the number of papers published over the last few years reflects the continued clinical desire for in vivo measures of metabolism and a renewed interest in the CCO signal following the demonstration of new technological and analytical methods.

Our focus in this review article is on measurements in patients and healthy volunteers, however, in order to fully understand the chronology and advances made in the methodology, some important preclinical work is also discussed. While there is extensive work on the measurement of CCO changes in the visual part of the light spectrum (see the review by LaManna for a historical perspective ${ }^{26}$ ), here, we have focused on the measurements with NIR light (650 to $1000 \mathrm{~nm})$. We aim to summarize work on the instrumentation, algorithms, and clinical applications of the CCO signal and assess the current state of the measurement to look forward to the future of the technique. We provide a simplified description of the biochemistry of the enzyme to understand the origins of the optical signal; the methods, algorithms, and instrumentation used to study it; a discussion of the issues with the methodology; a summary of the applications of cerebral NIRS measurements of CCO in neonatal and adult studies; and a forward perspective on the future of the technique. Almost 40 years from the advent of the first measurement of CCO using NIRS, this signal continues to hold significant interest and hope in providing the ultimate noninvasive, in vivo, bedside, and real-time sensor of brain tissue well-being; a sensor with the capacity to indicate when to alter and/or redirect therapy to improve long-term clinical outcome.

We are interested in an indicator of the redox state change of CCO. Previous NIRS studies, both preclinical and clinical, have referred to this signal as $\mathrm{CytOx}$, aa3, $\mathrm{Cu}_{\mathrm{A}}, \mathrm{CCO}$, and oxCCO. For clarity, in this review, the term oxCCO is used to describe the NIRS measurement of the oxidation state of cytochrome-coxidase, which arises from its redox state-dependent changes in the NIR spectrum. Although the dominant chromophore in the NIR is the CuA center, other cytochrome redox centers and oxygen intermediates can contribute to this signal.

\section{Biochemistry and Spectral Features of Cytochrome-C-Oxidase}

In order to fully understand the CCO NIRS signal, one must understand the biochemistry from which the signal stems. $\mathrm{CCO}$ is a small but important part of the aerobic metabolism of glucose. Glycolysis converts glucose into pyruvate and in the process generates ATP and NADH. After glycolysis, pyruvate is transported into the mitochondria and converted to acetyl CoA, which then enters the tricarboxylic acid (TCA) cycle. During the TCA cycle, additional ATP and NADH are produced. NADH is an electron donor; electrons from NADH can be used to, ultimately, convert oxygen to water, releasing useful chemical-free energy in the process. The mitochondrial electron transfer chain (ETC) mediates this transfer, in the process converting ADP to ATP. The consequent change in ATP/ ADP ratio provides usable energy to drive a wide variety of cellular processes. 


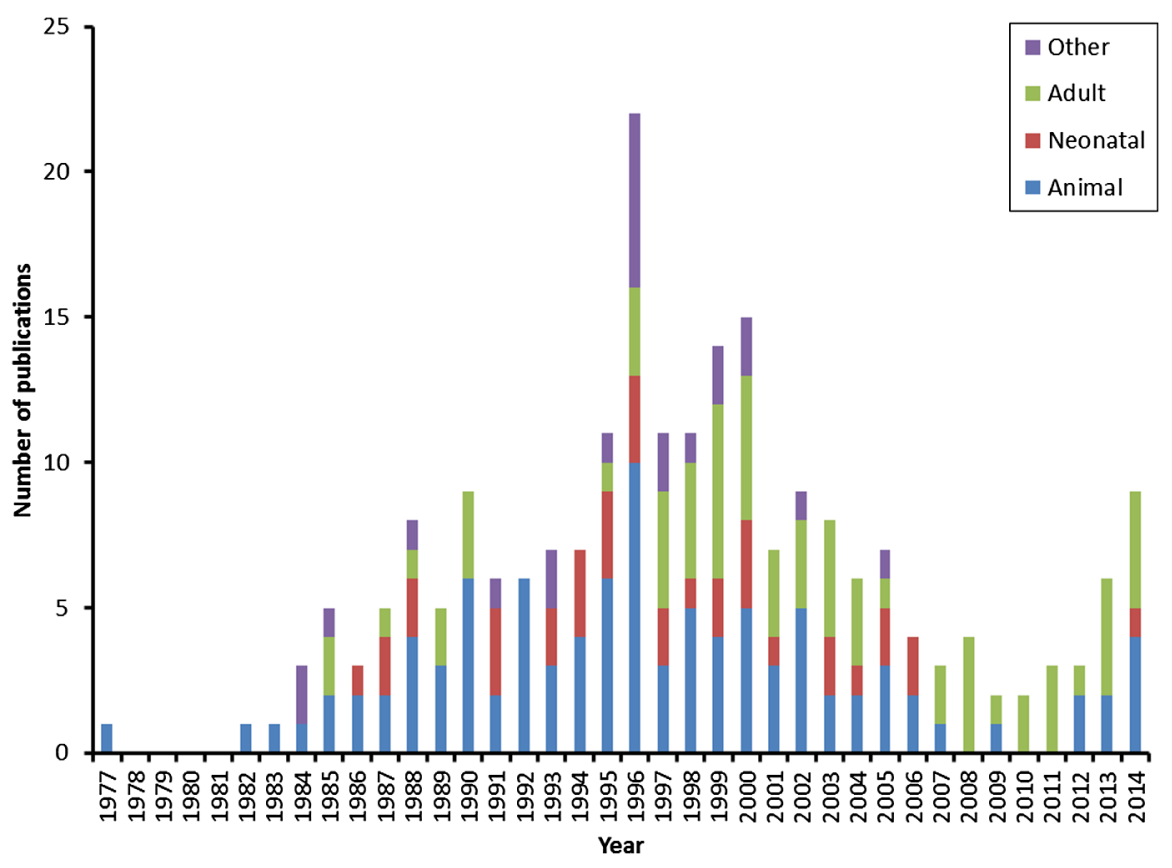

Fig. 2 Number of papers published per year on the subject of cerebral NIRS measurements of CCO. The data were taken from the PubMed results for "cytochrome oxidase infrared spectroscopy brain" (with the addition of the 1977 Jöbsis paper). There are a total of 223 papers split into categories based on the subject: adults-67 papers (green), neonates-36 papers (red), animals-98 papers (blue) and other-23 papers (purple, including reviews of the subject, in vitro work and computational simulations).

Figure 3 shows the mitochondrial protein complexes involved in this oxidative phosphorylation. Oxidative phosphorylation is the process where energy is harnessed through a series of protein complexes (known as complexes I to V) embedded in the inner membrane of mitochondria to create ATP. Oxidative phosphorylation can be broken down into two parts: oxidation of NADH (and other electron donors) in the ETC via complexes I to IV and phosphorylation, the production of ATP, at Complex V. Complex I accepts electrons from $\mathrm{NADH}$ and transfers them to the soluble membrane-bound electron carrier ubiquinol $(\mathrm{Q})$, producing ubiquinone $\left(\mathrm{QH}_{2}\right) \cdot \mathrm{QH}_{2}$ is oxidized by complex III, in the process reducing the soluble electron transport protein, cytochrome c. Cytochrome $\mathrm{c}$ is then oxidized by the terminal electron acceptor cytochromec-oxidase (also known as complex IV), with the electrons ultimately being used to reduce oxygen to water. The redox potential drop in the electron transfer processes in complex I, III, and IV is converted to a proton electrochemical potential, which ultimately drives mitochondrial ATP synthesis via the mitochondrial ATP synthase (complex V).

The transfer of electrons within the mitochondrial ETC complexes occurs via a number of protein bound redox factors. Of particular interest in this review, $\mathrm{CCO}$ contains four redox centers: two hems (known collectively as $\mathrm{aa}_{3}$ ) and two copper sites (see Fig. 3). The electrons pass between these centers in a series of redox reactions. All of these redox changes have associated optical transitions. In the NIR region, one of the copper sites, the $\mathrm{Cu}-\mathrm{Cu}$ dimer copper $\mathrm{A}\left(\mathrm{Cu}_{\mathrm{A}}\right)$, dominates the absorption spectrum, with a strong peak in the oxidized form centered, in mammalian enzymes, around 830 to $840 \mathrm{~nm}$ (see Fig. 4). For a recent detailed discussion of the relative contributions of CCO chromophores to the NIR spectrum, see Ref. 30 and references therein.

The difference between these absorption spectra (oxidizedreduced $\mathrm{CCO}$ ) can be used to monitor changes in the redox states of the mitochondrial proteins. Note that the total $\mathrm{CCO}$ concentration does not change over a short-time period (in the order of hours); therefore, for analytical purposes, it is only necessary to use the difference spectrum between the oxidized and reduced species to obtain an indicator of the changes in the $\mathrm{CCO}$ redox state.

The UCL group has shown that using the oxidized-reduced difference spectrum resolves changes in oxidized CCO (and conversely, using the reduced-oxidized difference spectrum resolves changes in reduced $\mathrm{CCO}$ ). The proof uses the modified Beer-Lambert law and involves two scenarios. In scenario 1 [Eqs. (1)-(5)], CCO is fully oxidized (ox) at the initial time $\left(t_{0}\right)$ and so the Beer-Lambert law states that the attenuation (A) due to $\mathrm{CCO}$ is related only to the concentration (c) of oxidized $\mathrm{CCO}$, its extinction coefficient $(\varepsilon)$, and the optical pathlength of light $(l)$ :

$A\left(t_{0}\right)=l \varepsilon_{\mathrm{oxCCO}} C_{\mathrm{oxCCO}}\left(t_{0}\right)$.

At a later time $\left(t_{1}\right)$, CCO is not fully oxidized and so the attenuation is related to both oxidized and reduced (red) forms of the enzyme:

$A\left(t_{1}\right)=l\left[\varepsilon_{\mathrm{oxCCO}} C_{\mathrm{oxCCO}}\left(t_{1}\right)+\varepsilon_{\mathrm{redCCO}} C_{\mathrm{redCCO}}\left(t_{1}\right)\right]$.

Assuming that the total CCO concentration does not change, the initial concentration of fully oxidized $\mathrm{CCO}$ is equal to the sum of the oxidized and reduced forms at the later time:

$C_{\mathrm{oxCCO}}\left(t_{0}\right)=C_{\mathrm{oxCCO}}\left(t_{1}\right)+C_{\mathrm{redCCO}}\left(t_{1}\right)$.

Therefore, it is possible to substitute the equations above into the difference measurement of the attenuation: 


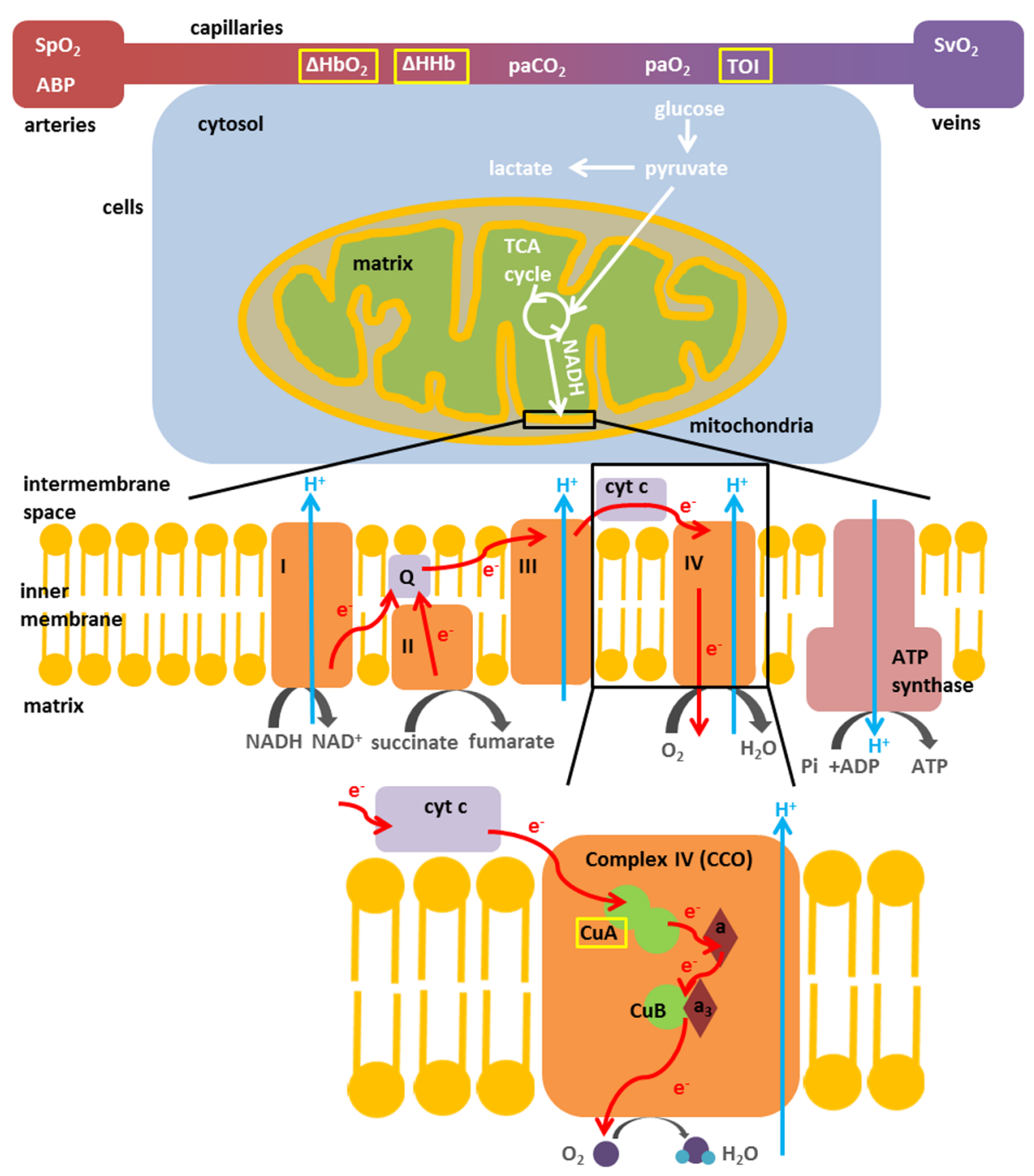

Fig. 3 Schematic of cellular metabolism with the electron transport chain and CCO expanded. CCO is represented as complex IV. This diagram is limited to the carbohydrate metabolism (from glucose); fat metabolism, also aerobic, is ignored. Chromophores that are measured by NIRS are highlighted in yellow boxes.

$$
\begin{aligned}
\Delta A= & A\left(t_{1}\right)-A\left(t_{0}\right) \\
= & l\left\{\varepsilon_{\mathrm{oxCCO}}\left[C_{\mathrm{oxCCO}}\left(t_{1}\right)-C_{\mathrm{oxCCO}}\left(t_{0}\right)\right]\right. \\
& \left.+\varepsilon_{\mathrm{redCCO}} C_{\mathrm{redCCO}}\left(t_{1}\right)\right\} \\
= & l\left\{\varepsilon_{\mathrm{oxCCO}}\left[C_{\mathrm{oxCCO}}\left(t_{1}\right)-C_{\mathrm{oxCCO}}\left(t_{0}\right)\right]\right. \\
& \left.+\varepsilon_{\mathrm{redCCO}}\left[C_{\mathrm{oxCCO}}\left(t_{1}\right)-C_{\mathrm{oxCCO}}\left(t_{0}\right)\right]\right\} \\
= & l\left(\varepsilon_{\mathrm{oxCCO}}-\varepsilon_{\mathrm{redCCO}}\right) \Delta C_{\mathrm{oxCCO}} .
\end{aligned}
$$

The oxidized-reduced difference (diff) extinction spectrum, $\varepsilon_{\text {diff }}=\varepsilon_{\text {oxCCO }}-\varepsilon_{\text {redCCO }}$, gives rise to Eq. (5), which expresses the change in attenuation due to a change in the concentration of oxidized $\mathrm{CCO}$ from the difference extinction spectra:

$$
\Delta A=l \varepsilon_{\text {diffCCO }} \Delta C_{\text {oxCCO }} .
$$

In a scenario 2 [Eqs. (6)-(10)], where CCO is not fully oxidized initially (i.e., CCO exists in both oxidized and reduced forms), the same expression can be achieved:

$$
A\left(t_{0}\right)=l\left[\varepsilon_{\text {oxCCO }} C_{\text {oxCCO }}\left(t_{0}\right)+\varepsilon_{\text {redCCO }} C_{\text {redCCO }}\left(t_{0}\right)\right],
$$

$A\left(t_{1}\right)=l\left[\varepsilon_{\mathrm{oxCCO}} C_{\mathrm{oxCCO}}\left(t_{1}\right)+\varepsilon_{\mathrm{redCCO}} C_{\mathrm{redCCO}}\left(t_{1}\right)\right]$.

Assuming again that the total CCO concentration does not change:

$$
C_{\text {oxCCO }}\left(t_{0}\right)+C_{\text {redCCO }}\left(t_{0}\right)=C_{\text {oxCCO }}\left(t_{1}\right)+C_{\text {redCCO }}\left(t_{1}\right) .
$$

Therefore,

$$
\begin{aligned}
\Delta A= & A\left(t_{1}\right)-A\left(t_{0}\right) \\
= & l\left[\varepsilon_{\mathrm{oxCCO}}\left(C_{\mathrm{oxCCO}}\left(t_{1}\right)-C_{\mathrm{oxCCO}}\left(t_{0}\right)\right)\right. \\
& \left.+\varepsilon_{\mathrm{redCCO}}\left(C_{\mathrm{redCCO}}\left(t_{1}\right)-C_{\mathrm{redCCO}}\left(t_{0}\right)\right)\right] \\
= & l\left[\varepsilon_{\mathrm{oxCCO}}\left(C_{\mathrm{oxCCO}}\left(t_{1}\right)-C_{\mathrm{oxCCO}}\left(t_{0}\right)\right)\right. \\
& \left.+\varepsilon_{\mathrm{redCCO}}\left(C_{\mathrm{oxCCO}}\left(t_{1}\right)-C_{\mathrm{oxCCO}}\left(t_{0}\right)\right)\right] \\
= & l\left(\varepsilon_{\mathrm{oxCCO}}-\varepsilon_{\mathrm{redCCO}}\right) \Delta C_{\mathrm{oxCCO}},
\end{aligned}
$$

which gives rise to the same expression as Eq. (5):

$\Delta A=l \varepsilon_{\text {diffCO }} \Delta C_{\text {oxCCO }}$. 

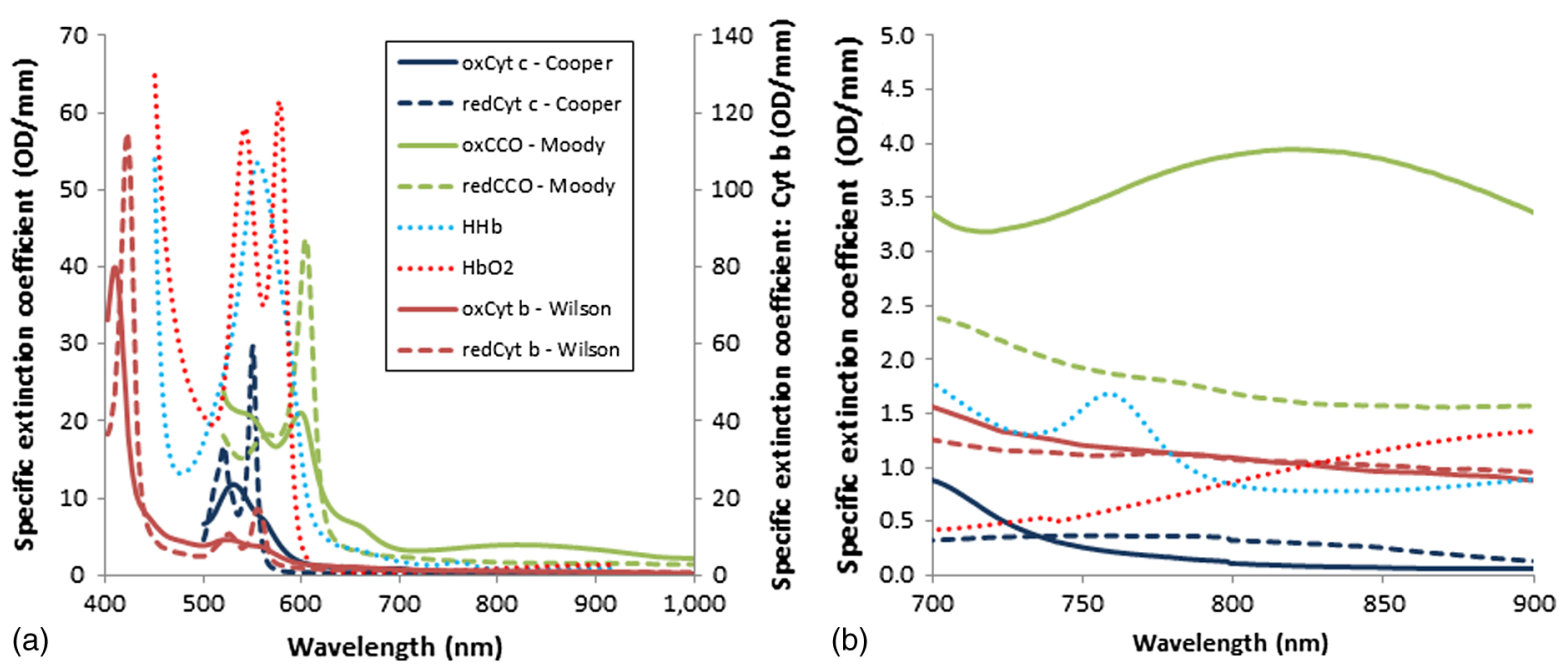

Fig. 4 The extinction spectra of the absorbing cytochromes in the electron transport chain in the visible and NIR. The hemoglobin species extinction spectra are included for reference (450 to $740 \mathrm{~nm}$ from Zijlstra et al. ${ }^{27}$ and 740 to $915 \mathrm{~nm}$ from Mark Cope, ${ }^{28}$ causing the discontinuity at $740 \mathrm{~nm}$ ). (a) Displayed between 400 and $1000 \mathrm{~nm}$, note that the cytochrome b spectra are plotted on a secondary axis and (b) zoomed into the NIR region of 700 to $900 \mathrm{~nm}$. Oxidized and reduced spectra of cytochrome c (oxCyt c and redCyt c, respectively) measured by Chris Cooper at the University of Essex in the porcine heart. Oxidized and reduced spectra of CCO (oxCCO and redCCO) measured by John Moody at the University of Plymouth in the bovine heart. Oxidized and reduced spectra of cytochrome b (oxCyt $b$ and redCyt b) measured by Mike Wilson at the University of Essex. All data taken from the UCL Biomedical Optics Research Laboratory (BORL) website in Ref. 29.

These proofs show that the oxidized-reduced difference spectrum can be used to resolve changes in the concentration of oxidized CCO. An increase in the oxCCO signal therefore reports an increase in oxidized $\mathrm{CCO}$ and an equivalent decrease in reduced $\mathrm{CCO}$. Note that if the sign of the measured signal is changed, it represents reduced $\mathrm{CCO}$, not oxidized CCO. The oxCCO term does not imply that we are only sensitive to the oxidized species. Nevertheless, oxCCO is the term used to describe the measured changes from the difference spectra in this review article.

To understand what a change in oxCCO means physiologically we need to know what the concentration of oxidized and reduced CCO is dependent on and how it can be affected in vivo. The redox state is dependent on both the availability of oxygen in the cells and the supply of electrons entering the ETC. The review by Cooper et al. ${ }^{31}$ gives an overview of the influencing factors on the CCO redox state. In the purified isolated enzyme, these include changes in the three substrates [protons $(\mathrm{pH})$, reduced cytochrome $\mathrm{c}$, and oxygen tension], as well as a range of terminal inhibitors (such as nitric oxide and cyanide) and electrochemical potential. The complex interplay between these factors in the mitochondrial ETC has been investigated mathematically by Banaji. ${ }^{32}$ This informs on clinical in vivo measurements. Possible factors that might increase oxCCO in vivo include increase in oxygen tension, increased ATP turnover, decrease in proton electrochemical gradient, decrease in $\mathrm{pH}$, or decrease in supply of reducing equivalents (substrates, i.e., NADH) to the ETC. These factors have been discussed by Heekeren et al. ${ }^{33}$

The oxCCO concentration can readily be decreased in animal models, either by large drops in oxygen tension or the addition of inhibitors that act at the oxygen reduction site. ${ }^{16}$ There have, though, been questions regarding how easy it is to increase the oxCCO signal in vivo, but animal models have shown that there is the capacity to increase the oxidation state at normoxia ${ }^{17,34}$ as well as adult volunteer studies in hyperoxia and hypercapnia, ${ }^{13,35}$ and functional activation. ${ }^{12,33}$ We acknowledge that there are some differences in the CCO response between the preclinical and clinical work, in particular, when compared to results from nonanesthetized human adults; it is not our intention in this review to discuss those physiological differences.

\section{Methods of Cytochrome-C-Oxidase Measurements}

$\mathrm{CCO}$ is one of the most abundant enzymes in mammalian systems. However, its relatively low concentration compared to the hemoglobin chromophores presents some challenges for NIRS techniques. The absolute concentration of $\mathrm{CCO}$ in the human brain is unknown, but optical methods have obtained values of 5.5 and $4.5 \mu \mathrm{M}$ in the adult rat. ${ }^{36,37}$ This should be considered an upper limit for the human CCO concentration as rats have a significantly higher cerebral metabolic rate of oxygen than humans. ${ }^{31}$ The contribution of $\mathrm{CCO}$ to overall absorption in tissue is considerably less than that of hemoglobin, because of its lower concentration, despite a higher specific extinction spectrum in the NIR region. Although the detectable absolute concentrations may vary with species, age, and optical set up, it is clear that the overall maximal signal from CCO should be $5 \%$ to $10 \%$ of that from hemoglobin. Therefore, light attenuation changes due to $\mathrm{CCO}$ can easily be masked by the much larger attenuation changes due to $\mathrm{HbO}_{2}$ and $\mathrm{HHb}$; this can result in cross talk. We define cross talk as a genuine change in one chromophore concentration inducing a spurious measured concentration change in another. Specific instrumentation and algorithms are necessary to be able to resolve these concentration changes without cross talk and we discuss this later in Sec. 3.4.

The principles of NIRS [only continuous wave NIRS (CW NIRS) is considered here] are based on calculating changes in concentration from the changes in the measured light attenuation in tissue. In order to do this, an algorithm and instrumentation 
must be chosen, along with the selection of other variables, such as the pathlength that the light has traveled, the absorption spectrum of the chromophore, and a set of measurement wavelengths. It is important that the algorithm and the other variables do not unknowingly induce artifacts. The factors that make up the algorithm and its variables have been the subject of discussion for many years. The choices to be made are:

- the algorithm

- the determination of the chromophore absorption spectra

- how the optical pathlength is estimated

- the number and choice of wavelengths.

Calculating and validating changes in hemoglobin in both of its oxygenation states is simpler because its concentration in tissue is high. It is also relatively simple to isolate the molecule to produce an absorption spectra in vitro. The absorption spectra have defined features in the NIR (see Fig. 5), such as a peak at 750 to $760 \mathrm{~nm}$ in $\mathrm{HHb}$ and the isobestic point at $800 \mathrm{~nm}$. The $\mathrm{CCO}$ difference absorption spectrum, once identified, has a broad peak, which is significantly different to that of the hemoglobin chromophores. However, the broad nature of the three chromophore peaks throughout the NIR region confounds simple deconvolution via standard optical techniques, such as dual wavelength or derivative spectroscopy. Therefore, to successfully separate the CCO signal from the larger hemoglobin signals, careful selection of the algorithm, wavelengths, and extinction spectra is required. One of the biggest problems for the oxCCO signal is that there is no gold standard with which to compare and validate the NIRS signal experimentally. Although the same problem exists for NIRS detectable hemoglobins, in this case, there are at least invasive measurements that can be assumed to report on similar parameters (such as tissue oxygen, blood volume, and arterial/venous oxygen saturation). The lack of external comparators is a problem in assessing which criteria produce the most physiologically accurate oxCCO signal. Yet, there have been a range of in vitro and in vivo controlled studies that inform on the success of the optical separation of the cellular oxCCO signal from changes in the hemoglobin signals. Additionally, integrated mathematical models of the biochemistry and physiology can aid the interpretation of results. ${ }^{38-40}$

\subsection{Algorithm}

Various algorithms have been developed by different groups, and these were assessed and described fully in 1995 by Matcher et al. ${ }^{41}$ The algorithms can be reduced to those developed at Duke University (Duke), University College London (UCL), Hokkaido University, Sapporo (Sapporo), and Keele University (Keele).

The UCL algorithm is a generalized algorithm based on the modified Beer-Lambert law and the concentrations are derived using multilinear regression. ${ }^{42}$ The modified Beer-Lambert law forms the mathematical basis of spectroscopic algorithms that relate wavelength-dependent optical attenuation signals to changes in chromophore concentrations. The generalized form of this algorithm for $n$ wavelengths (termed UCLn) is ${ }^{41}$

$$
\left[\begin{array}{c}
\Delta\left[\mathrm{HbO}_{2}\right] \\
\Delta[\mathrm{HHb}] \\
\Delta[\mathrm{oxCCO}]
\end{array}\right]=\frac{1}{\text { pathlength }}\left[\begin{array}{ccc}
\varepsilon_{\mathrm{HbO}_{2}}\left(\lambda_{1}\right) & \varepsilon_{\mathrm{HHb}}\left(\lambda_{1}\right) & \varepsilon_{\mathrm{oxCCO}}\left(\lambda_{1}\right) \\
\varepsilon_{\mathrm{HbO}_{2}}\left(\lambda_{2}\right) & \varepsilon_{\mathrm{HHb}}\left(\lambda_{2}\right) & \varepsilon_{\mathrm{oxCCO}}\left(\lambda_{2}\right) \\
\vdots & \vdots & \vdots \\
\varepsilon_{\mathrm{HbO}_{2}}\left(\lambda_{n}\right) & \varepsilon_{\mathrm{HHb}}\left(\lambda_{n}\right) & \varepsilon_{\mathrm{oxCCO}}\left(\lambda_{n}\right)
\end{array}\right]^{-1} \times\left[\begin{array}{c}
\Delta A\left(\lambda_{1}\right) \\
\Delta A\left(\lambda_{2}\right) \\
\vdots \\
\Delta A\left(\lambda_{n}\right)
\end{array}\right] .
$$

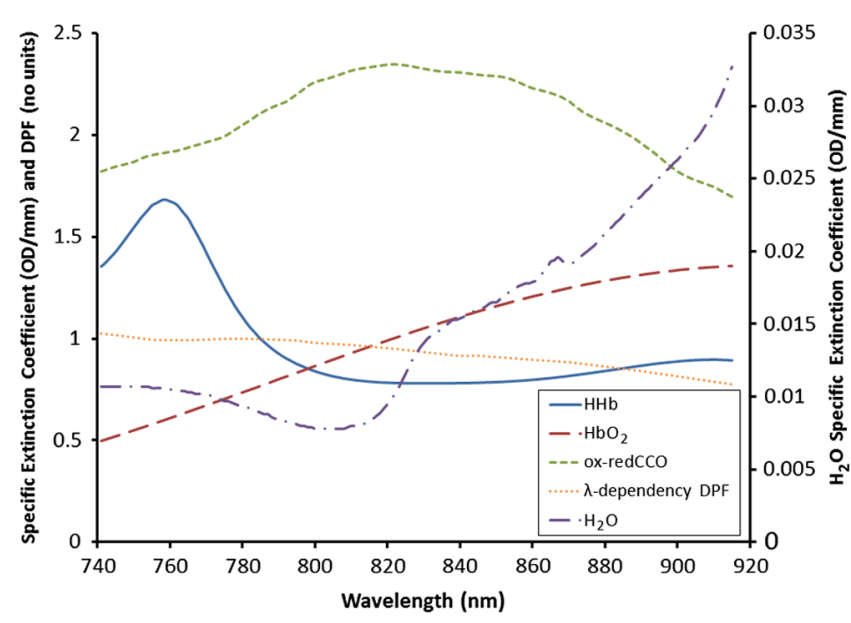

Fig. 5 Specific extinction coefficients measured by Mark Cope. ${ }^{28}$ The ox-redCCO spectra are not the same as the difference between the CCO spectra presented in Fig. 4. Data taken from the UCL BORL website in Ref. 29.
The algorithm assumes that tissue scattering and pathlength remain constant throughout the measurement period and is therefore suitable for all geometries and optical systems. It ignores, however, the multiple scattering effects including the nonlinear relationship between absorption and attenuation; ${ }^{43}$ this is discussed further in the Sec. 3.2. A three-wavelength version of this algorithm was also developed by researchers at Keele using different extinction spectra. ${ }^{44}$

The algorithm developed in Sapporo is a four-wavelength algorithm, which uses attenuation differences between three measuring wavelengths and a fourth reference wavelength. ${ }^{45}$ The reference wavelength was selected in a region in which, the authors claim, has zero attenuation from CCO. The algorithm was developed specifically for the intact rat head through a vertical plane from the roof of the mouth to the top of the skull. The coefficients used (not shown here but presented in Refs. 41 and 46) contain optical instrumentation factors so are only specific to one instrument. Their algorithm does not attempt to quantify the concentration changes in hemoglobins and oxCCO, and thus measurements are expressed in relative terms. ${ }^{46}$ The authors assert their algorithm to be robust to changes in scattering and optical pathlength changes during the measurement period. ${ }^{46}$ 
The Duke algorithm uses four wavelengths with specific extinction spectra determined in vivo from the intact cat brain to determine concentration changes. ${ }^{47}$ The algorithm is not displayed here but can be found in Ref. 41, where it is also discussed.

\subsubsection{Chromophore Absorption Spectra}

The difference between the oxidized and reduced form of $\mathrm{CCO}$ can be determined in many different environments, such as the isolated enzyme, in mitochondria, and in vivo. The first NIRS measurement used difference spectra that were taken through animal heads during anoxic challenges. ${ }^{1}$ These spectra showed clear differences between oxidized and reduced CCO states. Later, authors replaced blood with optically clear oxygen carrying perfluorocarbon solutions in an attempt to produce hemoglobin-free CCO spectra. ${ }^{18,21,48}$ In addition, later studies were able to remove this contribution completely in rats, ${ }^{49,50}$ cats, ${ }^{51-53}$ and piglets. ${ }^{16}$

The use of perfluorocarbon perfused blood-free animals has been a key to the development of algorithms to detect oxCCO. In some cases, these spectra were themselves used to generate the algorithms; in others, they were used to validate the use of in vitro spectra. The latter was the case for the UCL algorithms.

$\mathrm{Cope}^{28}$ found that there is no appreciable difference between the spectra measured in vivo and in vitro. Matcher et al.$^{41}$ found similar results when using the four-wavelength UCL algorithm, which uses in vitro extinction spectra, and the three-wavelength Keele algorithm, which uses in vitro spectra but with whole blood, not purified hemoglobin, to resolve concentration changes from the same attenuation data.

\subsection{Optical Pathlength}

The estimation of optical pathlength is one of the most notable challenges for the calculation of changes in oxCCO, as it is thought that erroneous changes resolved in oxCCO (i.e., cross talk) could be due to pathlength changes causing insufficient chromophore separation. ${ }^{16,23}$ As mentioned in Sec. 3.1, the modified Beer-Lambert law does not account for tissue scattering and assumes that the reduced scattering coefficient $\left(\mu_{\mathrm{s}}^{\prime}\right)$ is constant throughout a measurement period. The optical pathlength term is the only consideration of scattering in the equation, so it must be estimated sufficiently to produce physiologically accurate representations of chromophore changes in vivo.

The first attempts at optical pathlength estimation assumed a constant pathlength, equal to the product of the distance between the source and detector optodes and the differential pathlength factor (DPF), an experimentally determined factor to account for the increased pathlength of light due to all forms of scattering. ${ }^{42}$ The DPF can be determined using frequency-domain NIRS (FD NIRS) or time-resolved NIRS (TR NIRS), which estimate the optical pathlength. ${ }^{42,54,55}$ In addition, to monitor the changes in pathlength in real time with CW NIRS, Matcher et al. ${ }^{56}$ at UCL developed a method to resolve the pathlength using the water absorption spectrum and the measured broadband attenuation; features in the second differential of the water absorption spectra around 740 and $820 \mathrm{~nm}$ can be combined with an assumed tissue concentration of water to estimate pathlength.

In 1993, the modified Beer-Lambert law-based algorithm was updated to account for the wavelength-dependency of the DPF, which allowed for the decreasing scattering effects at increasing wavelengths in tissue.$^{57} \mathrm{DPF}$ varies with wavelength through another attenuation mechanism; high absorption of light at a specific wavelength decreases the likelihood that light at that wavelength will reach the detector.

A few experiments have been set up specifically to test whether changes in pathlength are responsible for cross talk in the oxCCO measurement; none of these studies found significant changes in the pathlength during events where changes in oxCCO were observed, such as hypoxemia ${ }^{58}$ or functional activation. ${ }^{12,59}$ Skov and Greisen ${ }^{60}$ suggest that the wavelength-dependent DPF is a solution to the cross talk problems; they observed a linear relationship between $\mathrm{HbT}$ and oxCCO during hypoxemia when using a constant DPF but not when using wavelength-dependent DPF.

There are physiological factors that will affect tissue scattering. For example, glucose levels affect the refractive index of extracellular fluid, which contributes to the reduced scattering coefficient; therefore, changes in the glucose concentration may affect the overall scattering of the tissue. ${ }^{61}$ Swelling of the brain, or edema, is likely to change cerebral scattering properties. There is therefore a need to understand the influence of changing tissue scattering properties on the calculation of chromophore concentrations or to measure scattering during the measurement period, particularly in cases such as brain injury.

\subsection{Wavelength Selection}

In theory, to extract information for three chromophores requires measurements at three wavelengths. Yet this approach is prone to noise and cross talk artifacts that may lead to inaccurate quantification of changes in concentrations and misinterpretation of data. When measurements at a larger number of wavelengths are used in spectroscopic algorithms, any detrimental effects of cross talk and noise are expected to diminish.

Due to the broad spectral peak in the oxidized-reduced CCO spectra and its relatively low concentration in vivo, the choice of selection of the specific wavelengths and number of wavelengths used for spectroscopy is a big factor. This is also relevant for the hemoglobin chromophores. Matcher et al. ${ }^{41}$ performed analysis to assess the separation of the oxCCO signal from the hemoglobin signals with different number of wavelengths $(4,6$, and " $n$ " or 112) in a range of data: rat head, piglet head, adult forearm, and a simulated dataset. The results showed that the higher number of wavelengths produced more accurate simulations and improved the in vivo measurements. Despite this, preclinical studies showed that limiting broadband measurements from 780 to $900 \mathrm{~nm}$ improves the resolution of oxCCO as exclusion of shorter wavelengths lessens the contribution of $\mathrm{HHb}$, which has a large peak at $760 \mathrm{~nm} .{ }^{16}$ Figure 6 shows graphically the selection of wavelengths used in the clinical papers mentioned in this review.

Broadband spectroscopy, offering a full range of measurement wavelengths, can be difficult to incorporate into wireless and multichannel systems. A recent study has used a genetic algorithm method ${ }^{102}$ to assess the minimum number of wavelengths needed to resolve oxCCO accurately (compared to the current gold standard of a broadband NIRS system across 780 to $900 \mathrm{~nm}$ at 1-nm wavelength resolution). ${ }^{62,103}$ The results show that the optimal combination of wavelengths is a set that almost evenly spans the spectral range (see Fig. 6). The error against the gold standard reduces as the number of wavelengths increases; increasing from 3 to 4,5 , and 8 wavelengths leads to a 


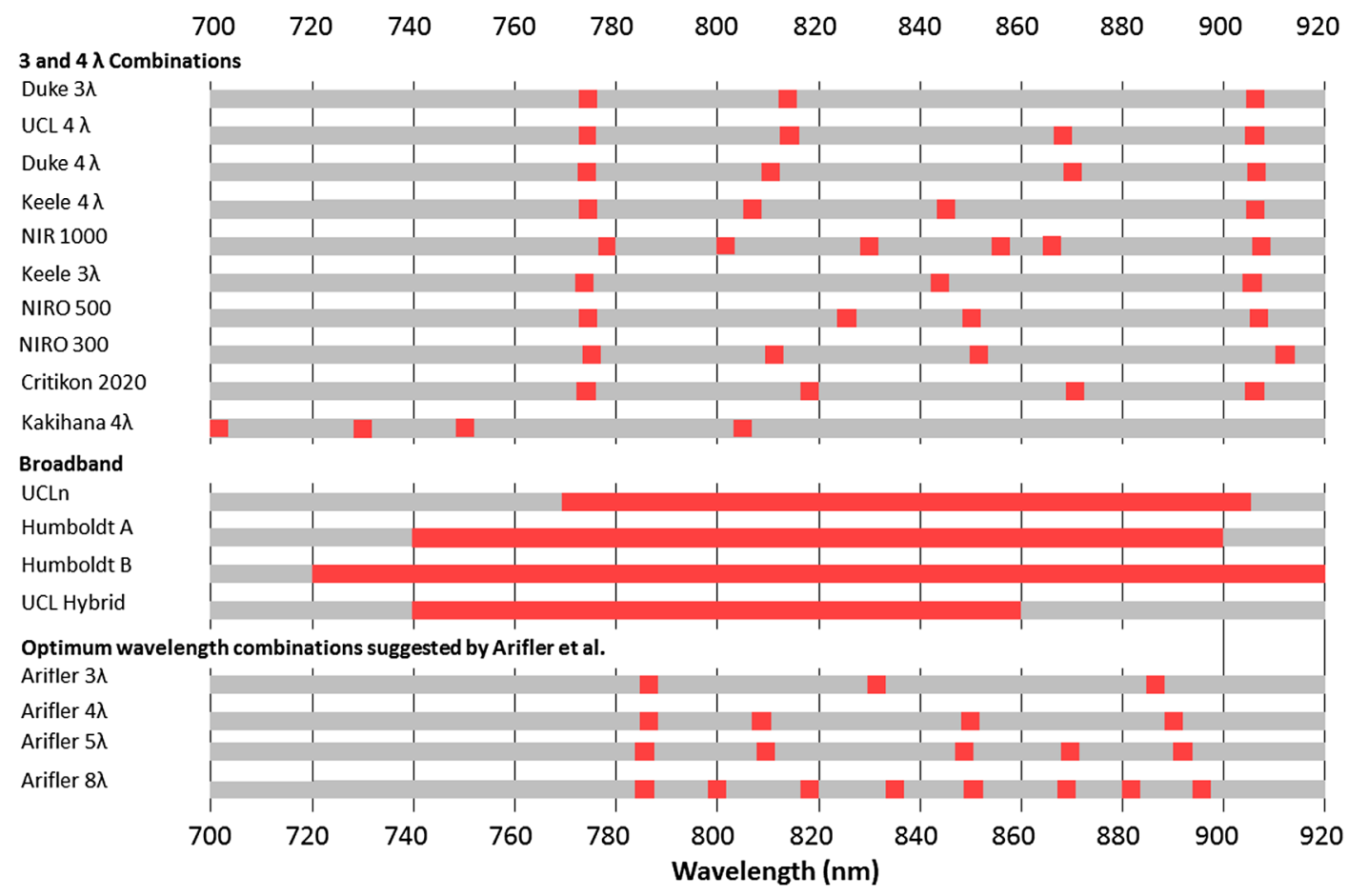

Fig. 6 Chart of wavelengths used in NIRS systems from all clinical publications mentioned in this review. Systems are listed in chronological order from their first publication in this review and are separated into three- and four-wavelength combinations and broadband spectra. The suggested wavelengths determined by Arifler et al. are also shown in Ref. 62. Clinical papers using the wavelengths are Duke $3 \lambda,{ }^{22,63,64}$ UCL $4 \lambda,{ }^{65}$ Duke $4 \lambda,{ }^{66-68}$ Keele $4 \lambda,{ }^{60,69-71}$ NIRO $1000,{ }^{72-75}$ Keele $3 \lambda,{ }^{76,77}$ NIRO $500,{ }^{78-85}$ NIRO $300,{ }^{86-93}$ Critikon 2020,94 UCLn, ${ }^{7,41,58,95,96}$ Humboldt $A{ }^{33,97}$ Humboldt $B,{ }^{98,99}$ and UCL Hybrid. ${ }^{12,13,59,100,101}$

two-, three-, and sevenfold improvement in the reduction of errors. The wavelengths selected need to be spread across this range in order to resolve the shape of the ox-redCCO spectra and disentangle it from the other chromophores; Arifler et al. ${ }^{62}$ found that the optimum eight wavelengths were 784, 800, 818, 835, 851, 868, 881, and $894 \mathrm{~nm}$ (displayed in Fig. 6).

\subsection{Optical Instrumentation}

Advances in optical methods have allowed further development of the instrumentation, as well as the algorithm, which have contributed to the increased robustness of the measured oxCCO signal with NIRS. For a full review of NIRS instrumentation, see Ref. 4.

Initially, NIRS instrumentation for the measurement of oxCCO was performed using three or four narrowband lasers at specific wavelengths between 740 and $910 \mathrm{~nm}^{22}$ (see Fig. 6, for more examples) using a photomultiplier tube (PMT) for detection. Commercial systems were developed to measure oxCCO using four discrete wavelengths using lasers and either photodiode detectors or PMTs (NIRO 300 and NIRO 500 respectively, Hamamatsu, Japan). ${ }^{82,86}$

In UCL, systems have increasingly been developed in-house and focused on broadband spectroscopy in the aim of obtaining a more robust oxCCO signal ${ }^{41}$ (see Sec. 3.4). These systems typically use a broadband white light source (i.e., a tungsten halogen bulb) with a spectrograph and charge-coupled device using optical fibers to transmit light both to and from the tissue. ${ }^{10,96,104}$ The UCL broadband NIRS systems include (1) a broadband instrument developed in the $1990 \mathrm{~s}$ by Springett et al.; ${ }^{104}$
(2) a multidistance hybrid broadband and FD system developed by Tachtsidis et al. in 2010 (called hybrid optical spectrometer or pHOS); $;^{25,101}$ and (3) in 2014, two variations of that system without the FD component developed by Bale et al. ${ }^{96}$ to be used in neonates (called Cytochrome Research Instrument and appLication or CYRIL),${ }^{96}$ and Phan et al. ${ }^{105}$ with the capacity to perform imaging. All UCL-developed systems are broadband instruments with the capacity to apply the UCLn algorithm in real time and are explicitly mentioned in the reported studies below.

Miniaturization is a trend seen in all forms of technology and NIRS is no exception. The trend toward making devices smaller, more portable and cheaper has pushed broadband spectroscopy toward miniature spectrometers, ${ }^{106}$ and discrete wavelength systems toward wireless NIRS. ${ }^{107}$ For wireless devices, in which there is a need to eliminate heavy fiber optics, LEDs, and laser diodes can be applied directly to the head with wireless transmitters to send the data to a nearby computer. Versions of both of these technologies are in development with the aim of incorporating a measurement of oxCCO. ${ }^{108}$

In 2000, a combined CW and FD NIRS system was developed to use measurements of $\mu_{\mathrm{s}}^{\prime}$ and the absorption coefficient $\left(\mu_{\mathrm{a}}\right)$ to inform the calculation of absolute chromophore concentrations. ${ }^{109}$ This was further developed by UCL to the pHOS system that attempted to measure oxCCO using the UCL hybrid algorithm. ${ }^{25,101}$ These hybrid devices use broadband $\mathrm{CW}$ systems and several lasers in the FD $\left(7^{109}\right.$ and $4^{25}$ wavelengths) to achieve this. By resolving for both absorption and scattering, it may be possible to measure absolute concentrations of the chromophores. 
It is important to ensure that the NIRS signals are sensitive to the brain, but it is not possible to measure this experimentally. Therefore, light propagation models have been used to estimate the sensitivity of NIRS measurements to the brain in the adult ${ }^{110,111}$ and neonate $e^{112-114}$ at different source-detector separations. Due to the different geometries and tissue densities in adults and neonates, there are differences in the propagation of light. $^{112}$ The cerebrospinal fluid (CSF) has a large positive impact on the sensitivity of absorption change in the brain, ${ }^{110}$ whereas the skull thickness has a negative impact. ${ }^{111}$ The thin skull and CSF layers in the neonate allow for more penetration of light into the cortex. ${ }^{112,113}$ Brigadoi et al. ${ }^{113}$ found that in neonates, a source-detector separation of $<2.14 \mathrm{~mm}$ has a brain sensitivity of $<5 \%$, which means that signals from a typical set up of $\sim 25$-mm separation will have a significant cerebral contribution; a source-detector separation of more than $8.4 \mathrm{~mm}$ will achieve more than $5 \%$ brain sensitivity in adults. In addition, computational modeling work from Fukui et al. ${ }^{112}$ suggest that while the white matter sensitivity in adults is almost negligible, even for large $(50 \mathrm{~mm})$ source-detector separations, the neonatal model shows high white matter sensitivity at as low as 10-mm separation. Furthermore, the CCO concentration in the brain is likely to change with maturity. Rodent studies have shown that there is an increase in the number of mitochondria per cell, as well as an increase in the number of mitochondrial proteins and respiratory enzymes per mitochondrion in the first postnatal days. ${ }^{115}$ Additionally, there is a difference in energy metabolism and functional activity in the rodent brain during postnatal development compared with the adult, and so this must be taken into consideration when monitoring $\mathrm{CCO}$ with NIRS.

\section{Analysis}

\subsection{Analysis of Broadband Cytochrome-C-Oxidase Measurements}

Here, we document clinical experiments that assess the oxCCO signal for robustness and independence from other chromophores (i.e., cross talk). We focus mainly on clinical papers that use many wavelengths (broadband NIRS) and the wavelength-dependent DPF methods, which are required to minimize cross talk. We present a strong weight of evidence against cross talk if a sufficient number of wavelengths are utilized.

The simplest way to assess cross talk is to compare the oxCCO signals with the $\mathrm{HbO}_{2}$ and $\mathrm{HHb}$, and this has been done in many different experiments. In the assessment of the low frequency oscillations during visual stimuli, there was a phase shift between the hemodynamics and the oxCCO signal. ${ }^{97}$ Additionally, the authors noted that the oxCCO recordings were "remarkably stable ... compared to the changes in the hemoglobins, making a simple cross talk rather improbable." Another functional activation study also showed differences in the oxCCO directional changes despite normal hemodynamic response functions. ${ }^{12}$ Furthermore, the same study confirmed that there was no relationship between oxCCO and the measured systemic changes. During blood pressure changes in orthostatic hypotension patients, oxCCO dropped despite different HbT directional responses. ${ }^{93}$ During spontaneous desaturation events in infants with hypoxic-ischemic encephalopathy (HIE), different directional changes were seen in oxCCO despite consistent decreases in $\mathrm{HbO}_{2}$ and increases in $\mathrm{HHb} .{ }^{96}$ As discussed in Sec. 3.2, no study, where oxCCO and pathlength have been measured simultaneously, has a correlation been found between their changes.

Uludag et al. ${ }^{98}$ found from simulations that incorrect estimation of the wavelength dependency of partial pathlength can contribute to cross talk in chromophores with low concentration. However, when this was investigated experimentally by studies in healthy adult volunteers using the Humboldt B broadband NIRS system, they found that the majority of oxCCO changes could not be explained by this effect. ${ }^{24}$

Wolf et al. ${ }^{94}$ investigated the changes in oxCCO in 22 preterm infants during slow oxygen saturation $\left(\mathrm{SpO}_{2}\right)$ changes caused by inspired $\mathrm{O}_{2}$ changes. ${ }^{94}$ Changes in the concentrations of $\mathrm{HbO}_{2}, \mathrm{HHb}$, and $\mathrm{HbT}$, induced by small and slow $\mathrm{O}_{2}$ changes, were inversely correlated to the measured changes in the oxidation state of oxCCO with a four-wavelength Critikon 2020.

The most compelling evidence of a unique metabolic signal comes from human studies in which simultaneously measured changes in metabolism have been made using other modalities. Increases in oxCCO during hyperoxia correspond with decreases in microdialysis measures of lactate/pyruvate ratio, which is a marker of anaerobic metabolism. ${ }^{7}$ Tisdall et al. ${ }^{116}$ saw a linear relationship between oxCCO and estimated oxygen delivery [measured via pulse oximetry and transcranial Doppler (TCD) ultrasound] during hypoxemia. This relationship was not seen between $\mathrm{HbD}$ and oxygen delivery.

Residual analysis enables an observation of the goodness of fit of the chromophore spectra to the measured attenuation. To see if the CCO difference spectrum is necessary to explain the measured attenuation, two- and three-chromophore fits can be performed with and without CCO. Heekeren et al. ${ }^{33}$ found that using the three-chromophore fit explains in vivo data better than the two-component fit. ${ }^{33}$ The residuals for two-component fit have the same shape as oxCCO, which suggests that a chromophore with a similar shape to oxCCO is needed to fully explain the spectra. Above $760 \mathrm{~nm}$, the three-component fit residuals were random. This result was also found in other broadband adult studies ${ }^{12,58}$ and a neonatal study ${ }^{96}$ —an example of this process is shown in Fig. 7.

\section{Neonatal Studies}

In this section, we review NIRS measures of oxCCO in neonates during spontaneous changes in systemic physiology (e.g., periods of hypoxia, increases in blood pressure, and so on), during surgery, with neonatal drug administration, and during functional activation.

\subsection{Hypoxia and Other Spontaneous Systemic Changes}

In 1985, Jöbsis's group published the first NIRS study on newborn infants. ${ }^{22}$ They observed a decrease in oxCCO during spontaneous oxygen desaturations in three preterm infants using the Duke three-wavelength system. Furthermore, the timing and magnitude of the oxCCO decrease depended on the preceding oxygenation state, the depth and length of desaturation event and presence of circulatory disorder. Another study in which eight preterm infants were monitored with the NIRO 1000 showed that small $\mathrm{SpO}_{2}$ changes did not alter oxCCO. ${ }^{117}$ To add to the heterogeneity of the oxCCO response, Wickramasinghe et al. ${ }^{118}$ saw an inconsistent (increases and decreases) and insignificant change in oxCCO in seven infants during the reduction of inspired oxygen fraction $\left(\mathrm{FiO}_{2} ; \mathrm{SpO}_{2}\right.$ 
(a)

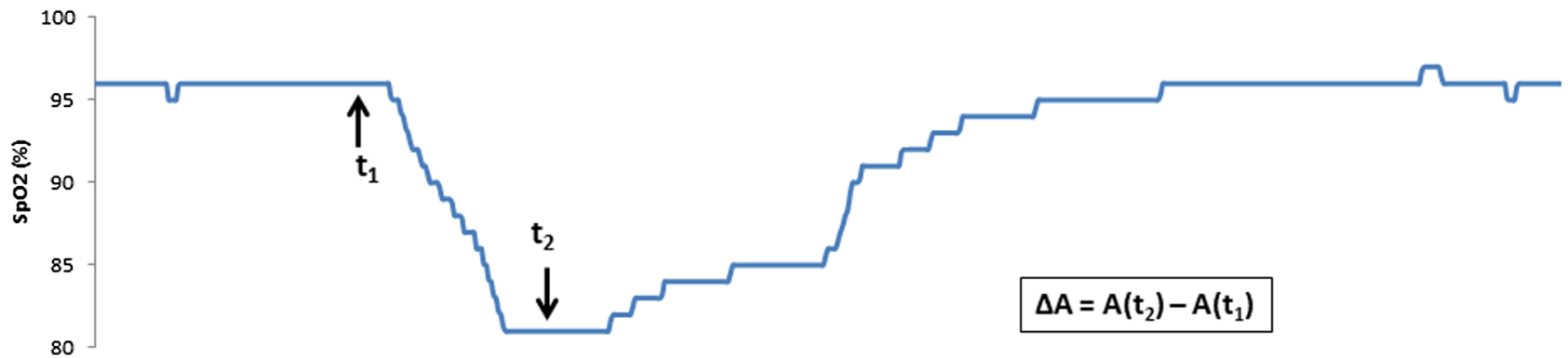

(b)

\begin{tabular}{|c|c|c|c|c|}
\hline Model & Pathlength & $\mathbf{\Delta H b O}_{\mathbf{2}}$ & $\mathbf{\Delta} \mathbf{H H b}$ & $\Delta \mathbf{o x C C O}$ \\
\hline $\mathbf{2}$ component & $14.97 \mathrm{~cm}$ & -1.19 & 1.49 & NA \\
\hline $\mathbf{3}$ component & $14.97 \mathrm{~cm}$ & -1.05 & 1.17 & -0.18 \\
\hline
\end{tabular}
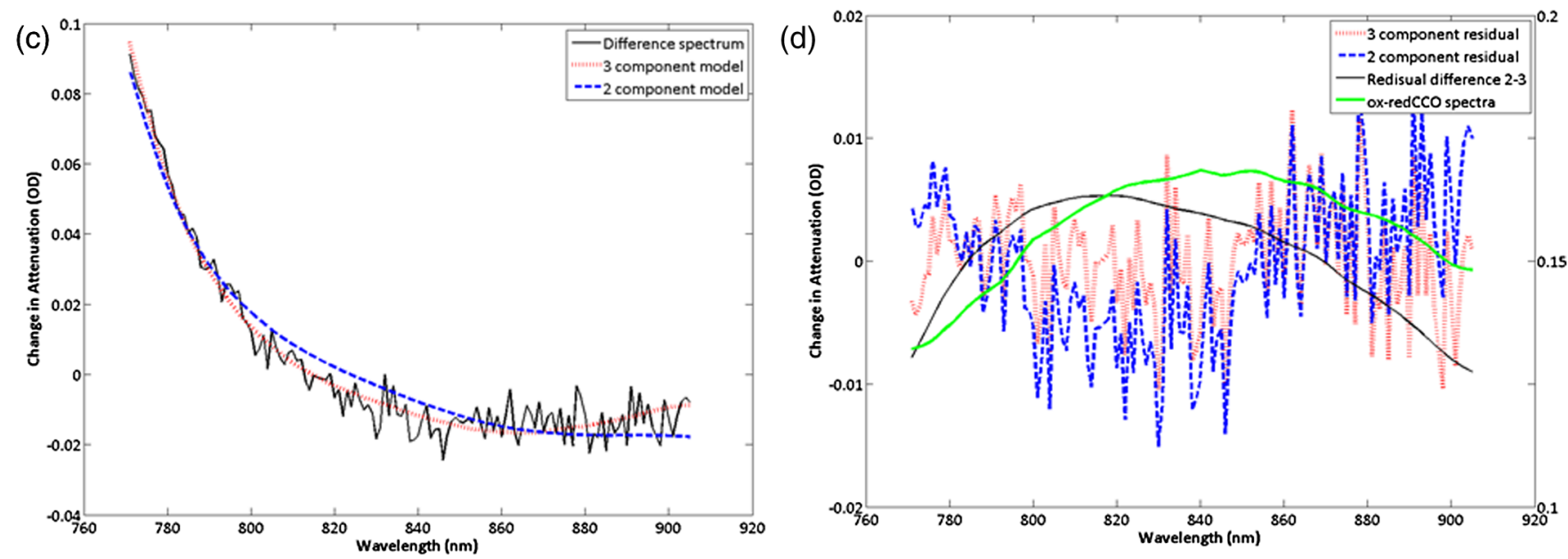

Fig. 7 Example residual analysis of NIRS data from the neonatal study by Bale et al. ${ }^{96}$ (a) $\mathrm{SpO}_{2}$ spontaneous decrease showing two time points, before and during hypoxia, from which the change in attenuation is calculated. (b) Concentration results from fitting change in attenuation spectra to two- $(\mathrm{HHb}$ and $\mathrm{HbO}_{2}$ ) and three- $\left(\mathrm{HHb}, \mathrm{HbO}_{2}\right.$, and ox-redCCO) component models. (c) Attenuation measured and attenuation fit back-calculated from two- and three-chromophore fits. (d) Residual differences between real attenuation and back-calculated attenuations, and the difference between them; note the shape of the difference between the residuals for the two- and three-chromophore fits is similar to the ox-redCCO spectra (also plotted for comparison on a separate scale).

change $\sim 10 \%$ ) using a three-wavelength Keele system. ${ }^{118} \mathrm{~A}$ study of infants during repetitive apneas found a consistent trend of reduced oxCCO during the breathing cycle. ${ }^{80}$ More recently, a UCL broadband NIRS study (with CYRIL) of over 200 oxygen desaturation events in six term infants with HIE saw changes in the concentration of oxCCO in both directions but with a decrease in oxCCO on average. ${ }^{96}$ The heterogenic response to these hypoxias may be a reflection of the level of brain injury, prematurity, previous oxygenation levels or influence of systemic pathophysiology. This heterogeneity is not seen in adult broadband NIRS studies of controlled hypoxias ${ }^{12}$ (see Sec. 6.1) so is specific to the neonatal brain. Animal work during anoxic swings in piglets has shown that as the oxygen delivery rate is reduced, there is a linear decrease in $\mathrm{HbO}_{2}$ but a biphasic elbow in oxCCO reduction. ${ }^{104} \mathrm{~A}$ reduction in oxCCO may be a signal of impending damage, and this has also been previously suggested by Cooper et al. ${ }^{31}$ The heterogeneity of the oxCCO response to hypoxia has also been seen in a piglet study of increasingly severe episodes of hypoxia. ${ }^{11}$ At lower severities of hypoxia $\left(8\right.$ and $\left.12 \% \mathrm{FiO}_{2}\right)$, there were small increases in oxCCO, while at higher degrees of hypoxia $(6 \%$, $4 \%$, and $0 \% \mathrm{FiO}_{2}$ ), oxCCO decreased with severity; consistent decreases in $\mathrm{HbO}_{2}$ and corresponding increase in $\mathrm{HHb}$ were observed in all hypoxic episodes. In 1985, Brazy called for an investigation into the degree of $\mathrm{CCO}$ reduction that causes brain injury in order to protect those at risk of injury; ${ }^{22} 30$ years later, there is still no answer but there is renewed effort to find it. ${ }^{96}$

Following from the hypoxia work, the effect of respiratory changes, which cause a subsequent change in carbon dioxide tension $\left(\mathrm{PaCO}_{2}\right)$, on the cerebral metabolism was investigated in neonates. The 1985 infant study from Brazy et al. ${ }^{22}$ saw oxCCO increase during hyperventilation; this was the first human study to suggest that oxCCO is not maximally oxidized in vivo at normoxia and can be increased by hyperventilation. The ability to increase oxCCO from normoxia is supported by another investigation where an increase in oxCCO was observed during an increase in $\mathrm{PaCO}_{2} \cdot{ }^{71}$ Edwards et al. ${ }^{117}$ suggest that the strong relationship between $\mathrm{PaCO}_{2}$ and oxCCO is either due to cerebral hemodynamic changes caused by vasodilation due to 
$\mathrm{CO}_{2}$, or $\mathrm{CO}_{2}$ decreasing the $\mathrm{pH}$ in cells. However, not all studies have seen this strong correlation; a study of $\mathrm{PaCO}_{2}$ changes in 24 ventilated preterm infants observed changes in cerebral blood flow and volume (CBF and $\mathrm{CBV}$, respectively), but not in oxCCO (four-wavelength Keele NIRS system). ${ }^{71}$ Brazy $^{64}$ reported the effects of crying on oxCCO and found different responses when lung disease was present and at different ages of infant. ${ }^{64}$ An insignificant small decrease was observed in oxCCO during endotracheal suctioning of 15 preterm infants in the presence of marked decrease in $\mathrm{HbO}_{2}$ and increase in $\mathrm{HHb}$, monitored with the NIRO $500 .^{82}$

Another early NIRS experiment examined changes in oxCCO during alterations in mean arterial blood pressure (MABP). ${ }^{63}$ This study showed that increases in MABP were accompanied by increases in $\mathrm{HbT}$ and $\mathrm{HHb}$ and a decrease in oxCCO as measured by a three-wavelength Duke system. Infants whose clinical condition did not improve over the study duration showed larger changes in $\mathrm{HbT}$ and oxCCO. This area has potential for further investigation, especially in terms of cerebral autoregulatory effects on tissue metabolism.

The effects of changing intracranial pressure (ICP) was investigated during lumbar puncture ${ }^{75}$ in six infants with posthemorrhagic ventricular dilation with the NIRO 1000; three infants had an increase in ICP, which was accompanied by an increase in oxCCO, the other three had no change in either ICP nor oxCCO; this is the only study to link oxCCO to ICP. In a 2004 study of 12 infants with intraventricular hemorrhage, removal of CSF, which is expected to cause a reduction of ICP, did not produce a change in oxCCO measured with the NIRO $500 .^{83}$

\subsection{Surgery and Anesthesia}

Concern for cerebral health during major surgery such as cardiopulmonary bypass (CPB) brought the introduction of NIRS to the operating room. The first use of oxCCO monitoring during neonatal surgery was to monitor cerebral metabolism throughout cardiac surgery. In 1991, 15 children were monitored during $\mathrm{CPB}$ at Duke with a four-wavelength system ${ }^{66}$ and a decrease of oxCCO was observed at onset of bypass with hypothermia and reduced further when rewarming began. In 1995, 63 infants were monitored during CPB and hypothermia, and interestingly, oxCCO decreases were observed despite $\mathrm{HbO}_{2}$ increases measured by the NIRO 500. ${ }^{85}$ Recovery of oxCCO was delayed despite $\mathrm{HbO}_{2}$ recovery to above baseline levels. Larger and more rapid decreases were seen in oxCCO in older ( $>14$ days) infants and those infants had worse recovery. The divergence in intravascular and mitochondrial oxygenation suggests abnormal response, which the authors suggested could be due to hypothermic temperature, effects of $\mathrm{pH}$ on the ETC, a disturbance in oxygen delivery to the mitochondria, disturbed perfusion, or decreased $\mathrm{HbO}_{2}$ dissociation. The delay in recovery suggests mitochondrial dysfunction. Changes in hemodynamics and oxCCO measured by a four-wavelength Keele system were seen in 5 out of 14 children, who were hypoxemic during CPB for congenital heart disease. ${ }^{60}$ Some recent studies with the NIRO 300 during repair of coarctation of aorta (without $\mathrm{CPB})^{87}$ and cardiac surgery ${ }^{119}$ showed no significant change in oxCCO throughout either procedure.

Other strains during surgery such as the use of therapeutic hypothermia and anesthesia will have effects on cerebral metabolism; such effects are interesting and important to understand if NIRS monitoring of metabolism is to become clinically relevant. However, as Du Plessis et al. ${ }^{85}$ note, it is difficult to disentangle the effects of hypothermia on oxCCO from the pathology or the stress of surgery.

\subsection{Effects of Drugs on the Neonatal Brain}

The effects of the anesthetic, propofol, during CPB in children $(n=11)$ and CPB controls without propofol administration $(n=13)$ were studied in $2000 .^{81}$ A decrease in oxCCO was observed during CPB and a lower decrease was seen in those with propofol.

The oxCCO response to other neonatal drugs, such as aminophylline, has been monitored. A NIRO 1000 was used to assess aminophylline delivery to 13 preterm infants in order to wean them off ventilators. ${ }^{74}$ A decrease in oxCCO was seen after administration, which the authors suggest could be mediated by reduction in perfusion of $\mathrm{CO}_{2}$. A later similar study, using a NIRO 300 on the effects of aminophylline and caffeine for treatment of apneas in preterm infants, ${ }^{89}$ found that neither drug made a significant change to oxCCO.

Measures of oxCCO have been used to identify cerebral metabolic changes caused by indomethacin for the treatment of patent ductus arteriosus. Despite using different NIRS systems, three separate studies recorded decreases in oxCCO, $\mathrm{HbO}_{2}$, $\mathrm{CBV}$, and $\mathrm{CBF}$ with increases in $\mathrm{HHb}$ when indomethacin was administered..$^{70,73,77}$ This result, combined with other evidence from changes in $\mathrm{CBF}$, has provided evidence against the use of the drug due to its reduction in $\mathrm{CBF}$ and cerebral oxygen consumption, in favor of other drugs, such as ibuprofen, which do not have such a cerebral metabolic effect. ${ }^{120}$

\subsection{Neonatal Brain Injury}

In 1997, a review by Cooper and Springett ${ }^{23}$ highlighted the potential for NIRS-measured oxCCO to monitor the progression of neonatal HI brain injury. The management strategy of HIE could be improved with additional information regarding the cerebral metabolic state as the development of the injury progresses over time and can lead to a secondary energy failure (SEF). There have been a handful of clinical studies of HIE with oxCCO which have promising results. A study of 11 infants with $\mathrm{HIE}$ showed that increases of $\mathrm{SpO}_{2}$ and $\mathrm{PaCO}_{2}$ increased oxCCO (measured with the UCL4 algorithm), which is a normal response and does not suggest pathophysiology. ${ }^{121}$ van Bel et al. ${ }^{69}$ saw a CBV and oxCCO (using the Keele four-wavelength algorithm) decrease in the first $12 \mathrm{~h}$ after birth in severe HIE, but stable changes in mild HIE and controls; this could suggest the delayed neuronal cell death as predicted by the animal models. They also saw a positive relationship between $\mathrm{PaCO}_{2}$ and oxCCO in all HIE infants.

Recently, the relationship between the UCLn NIRS-measured oxCCO and oxygen delivery $(\mathrm{HbD})$ was investigated in six HIE infants and found that an increased affinity between cerebral metabolism and oxygenation indicated brain injury. ${ }^{96}$ Analysis of the rewarming data after therapeutic hypothermia from this cohort provides more evidence that the relationship between the multimodal signals is indicative of brain injury. ${ }^{122}$ Further analysis of this data with a multivariate statistical technique showed that a strong relationship between oxCCO and the systemic physiology indicated more severe brain injury (as measured by ${ }^{31} \mathrm{P}$ MRS). ${ }^{123}$ This suggests that the importance of the oxCCO measurement may not lie in the signal itself, but in its relationship with oxygenation or 
systemic physiology, which can inform on the delicate equilibrium in pathology. However, most of this study occurred during therapeutic hypothermia, now a routine treatment for HIE, so it is difficult to compare with other earlier studies.

Furthermore, therapeutic hypothermia may make it difficult to observe SEF and cause unusual metabolic responses, as mentioned before. ${ }^{85}$ As previously discussed, there need to be more studies of the oxCCO response during cooling.

Recent animal studies of HIE have shown promising results. The UCL group demonstrated a relationship between broadband NIRS-measured oxCCO recovery fraction and HIE outcome in a piglet model. ${ }^{10}$

The oxCCO signal has recently been observed during neonatal stroke in a term infant for the first time. ${ }^{124}$ Repeated transient decreases in cerebral $\mathrm{HbT}, \mathrm{HbD}$, and oxCCO were noted on both cerebral hemispheres without significant changes in the monitored systemic physiology. A clear asymmetry was noted in the degree of change between the two sides of the brain. Cerebral oxygenation (measured with $\mathrm{HbD}$ ) and oxCCO were only highly coupled on the injured side of the brain.

\subsection{Functional Activation}

There has been a huge wealth of work in fNIRS using $\mathrm{HbO}_{2}$ and $\mathrm{HHb}$ as indicators of the hemodynamic response to neuronal activity since it was first presented in $1993 .{ }^{125}$ We have an opportunity with the oxCCO signal to inform directly on cerebral metabolism during neurovascular coupling; there is no other noninvasive, in vivo modality that can assess oxygen consumption during functional tasks. Concerns have been raised regarding the likelihood of measuring false positives or negatives in the hemodynamic response using fNIRS from hemodynamic signals that do not originate from neurovascular coupling. ${ }^{14}$ Given that the oxCCO signal is more brain specific than the hemodynamic signals, it could prove to be a very valuable addition to the fNIRS toolbox..$^{12,13}$

There has only been one functional activation study involving infants and oxCCO and this used the NIRO 300. Nineteen newborns were given auditory stimulus and no significant oxCCO change was observed despite a normal hemodynamic response. ${ }^{86}$ The expected response of oxCCO during functional activation is unknown, although an increase is often seen in adult studies of functional activation (see Sec. 6.2). Differences between the mature and neonatal brain are supported by the NIRS study during cardiac surgery by Du Plessis et al., ${ }^{85}$ where different oxCCO responses were seen at different ages. This could either reflect greater mitochondrial hypoxic tolerance in younger infants or differences in the concentration of $\mathrm{CCO}$ at different ages. ${ }^{85}$

\section{Adult Studies}

In order to characterize measured changes in the oxCCO signal, an increasing number of studies on adults have been performed over the past 10 years. These studies fall into three categories: volunteer studies with controlled inspired gas challenges to investigate the oxCCO response to systemic physiology, functional activation studies on healthy volunteers, and clinical studies.

\subsection{Volunteer Studies: Systemic Challenges}

The first NIRS studies on adult volunteers were performed in the early 1990s at Duke University. Hypoxic challenges were used to assess the changes in oxCCO in the adult brain at normocapnia ${ }^{67}$ and hyper- and hypocapnia ${ }^{68}$ with four-wavelength Duke NIRS systems. This work demonstrated a reduction of oxCCO during hypoxia at all levels of $\mathrm{PaCO}_{2}$.

More recently, the UCL group has performed hypoxemia studies $\left(\mathrm{SpO}_{2}\right.$ to $\left.80 \%\right)$ during normocapnia in healthy volunteers using the UCLn algorithm to estimate oxCCO. ${ }^{16,126}$ The hypothesis was that in nonanesthetized humans, a significant decrease in oxygen saturation should reduce oxCCO; this was confirmed in the study. There was a linear correlation between oxygen delivery [estimated from the measurement of the velocity of the middle cerebral artery (Vmca), an indicator of changes in CBF as monitored with TCD ultrasound] and oxCCO. A temporal delay between the $\mathrm{HbD}$ drop and oxCCO of $\sim 5 \mathrm{~s}$ was also observed. A later study using the pHOS and the UCLn algorithm confirmed the decrease in oxCCO during hypoxia. ${ }^{13}$

Conversely, in hyperoxia, the oxCCO signal has been found to increase. ${ }^{13,35}$ Tachtsidis et al. ${ }^{35}$ found that the oxCCO signal as measured with the UCLn algorithm was correlated with TOI (measured with the NIRO 300), as well as Vmca. Later, Kolyva et al. ${ }^{13}$ repeated this study using the pHOS and also showed that the magnitude of the oxCCO decrease during hypoxia was dependent on the distance between the source and the detector.

The oxCCO response to changes in $\mathrm{PaCO}_{2}$ was investigated first in 2009. ${ }^{35}$ During hypercapnia, Tachtsidis et al. ${ }^{35}$ increased inspired $\mathrm{CO}_{2}$ and saw a significant increase in oxCCO (estimated with the UCLn algorithm) and Vmca from baseline. There was no correlation between oxCCO and Vmca, but there were correlations with oxCCO and TOI (as measured with the NIRO 300). More recently, Kolyva et al. ${ }^{13}$ confirmed the increase in oxCCO during hypercapnia when monitored with the UCL pHOS system. They also performed hypocapnia and saw a decrease in oxCCO. Increased $\mathrm{CO}_{2}$ in the blood has a vasodilatory effect, which can increase $\mathrm{CBF}$, but it also decreases the $\mathrm{pH}$, which can reduce oxCCO. The authors suggested that the increases in blood flow from hypercapnia outweigh the potential damage to the respiratory chain from acidosis. In these studies, the oxCCO signal had a different trend to changes in HbT but showed a similar trend to TOI. A hyperventilation experiment from a different group also showed minor decreases in oxCCO with the NIRO $500 .^{78}$

As well as answering physiological questions, the study by Kolyva et al. ${ }^{13}$ was also designed to address scattering issues and brain-specificity questions using the UCL pHOS system. The magnitude of the oxCCO response increased with detector distance, which was not true for the hemoglobin signals. This oxCCO depth dependence shows that the signal is more brain specific, which is due to the higher $\mathrm{CCO}$ concentration in the brain, so the signal is less prone to extracerebral contamination than $\mathrm{HbO}_{2}$ and $\mathrm{HHb}$. Neither this study ${ }^{13}$ nor the previous one $^{116}$ saw any changes in pathlength during any of these physiological challenges.

\subsection{Volunteer Studies: Functional Activation}

Functional activation in adults has been studied with fNIRS since 1993 (see Sec. 5.5). Visual stimulation of the occipital cortex provides a robust stimulation method. There have been a few studies in this area to evaluate transient changes in the cellular energy metabolism that occur during changes in neuronal activity. Heekeren et al. ${ }^{33}$ saw an oxCCO increase during functional activation with a broadband NIRS (Humboldt A system); they speculate that the cause of the increased oxCCO is not due to increase in oxygen, as it would imply that the tissue is in a low 
$\mathrm{PaO}_{2}$ state normally, but due to decreases in proton electrochemical gradient increasing the rate of electron flow. ${ }^{33}$ This phenomenon was also seen by the same group in later papers with the Humboldt B broadband system. ${ }^{24,99}$ Fourier analysis of the frequencies showed that oxCCO and $\mathrm{HHb}$ signals have the same frequency as the stimulation, whereas $\mathrm{HbO}_{2}$ has other peaks, which can be due to other factors. ${ }^{99}$

Significant heterogeneous oxCCO responses were seen in an anagram-solving working memory study using the UCL pHOS system. ${ }^{12,100}$ No group significant change in oxCCO was seen during the anagram-solving task, but there were significant increases and decreases seen in different subjects. In some subjects, a significant decrease in oxCCO was seen in the presence of a typical hemodynamic response (increase in $\mathrm{HbO}_{2}$ and decrease in $\mathrm{HHb}){ }^{12,127}$ It is hard to predict or model the effect of functional activation on oxCCO; the authors suggested that the NADH oxidation and proton motive force can affect oxCCO without affecting hemodynamics, which may provide an explanation as to the heterogeneity of the oxCCO response despite a consistent homogenous hemodynamic reaction. This would support the theory of Heereken et al., ${ }^{33}$ which suggests a decrease in supply of reducing equivalents (substrates, i.e., $\mathrm{NADH}$ ) to the ETC that might alter oxCCO without affecting the hemoglobins.

Different depths give different oxCCO responses, which could be explained by spatial distribution of CCO, which is more highly concentrated in the brain than in the extracerebral tissue. $^{12} \mathrm{~A}$ recent working-memory study with CYRIL at multiple source-detector distances showed that the increase in oxCCO observed in the longer channels was not present in the short separation channels, despite a typical hemodynamic response in all channels. ${ }^{128}$ The addition of the measurement of the brain-specific oxCCO signal in fNIRS studies could help identify false positives and negatives and solve the problem of surface contamination.

\subsection{Clinical Studies}

Monitoring cerebral dysfunction in a clinical setting is important both in cases where the brain is at risk of injury (e.g., cardiac surgery) or where brain injury has already occurred [e.g., traumatic brain injury (TBI)]. There have been an increasing number of clinical NIRS studies in adults for pathologies, such as obstructive sleep apnea (OSA), cardiac surgery and hypotension, as well as brain injury itself.

The first investigation of cerebral oxCCO with NIRS during surgery occurred in 1995 due to concerns regarding cerebral stress of cardiac surgery causing postoperative neuropsychological damage. A NIRO 500 study of 41 patients undergoing cardiac surgery with CPB found that patients who suffered from neurological defects after surgery had lower minimum oxCCO values during surgery compared to those without defects. ${ }^{129}$ The oxCCO and venous saturation were inversely correlated during $\mathrm{CPB}$ suggesting that an increase in oxygen saturation may not be representative of an increase in tissue oxygenation. Kakihana et al. ${ }^{130}$ using the Sapporo algorithm and instrument saw that their measurement of oxCCO was a good predictor of postoperative cerebral outcome in a group of 66 patients who underwent thoracic aortic surgery; the recovery fraction of broadband NIRS-measured oxCCO following the surgery was predictive of neurological outcome.

Further, in cardiac surgery investigators recorded changes in oxCCO with the NIRO 300 during implantation of subpectoral implantable cardioverter defibrillators under general anesthesia. ${ }^{91}$ A random change in both directions was seen in oxCCO (measured with the NIRO 300) during implantable cardioverter defibrillator testing, despite consistent decrease in $\mathrm{HbO}_{2}$ and increase in $\mathrm{HHb}$. This suggests that the availability of oxygen at mitochondrial level was not consistently affected by a short term lack of oxygen delivery and that mitochondrial function may be able to identify cerebral abnormalities in this group of patients that hemodynamic changes do not reveal. A very early study also came to this conclusion, ${ }^{131}$ in patients who were candidates for carotid endarterectomy, carotid artery compression tests were performed and a reduction of oxCCO was seen in some, but not all, patients. The authors suggest that the presence of a decrease in oxCCO is indicative of a more severe impairment of brain function.

A case study of an adult woman with a long-term neurological disorder examined the oxCCO response to seizures with the NIRO $500 .{ }^{79}$ Prior to seizure activity, a gradual increase in $\mathrm{HbO}_{2}$ and $\mathrm{HbT}$ was observed with a simultaneous reduction in oxCCO. Throughout the seizure there was an increase in oxCCO, and $\mathrm{HHb}$; seizures cause increased metabolic demand and the increase in oxCCO corroborates this.

To investigate the response to hyperoxia in the brain injury, eight patients with TBI undertook a hyperoxia challenge $(60 \%$ and $\left.100 \% \mathrm{FiO}_{2}\right){ }^{7}$ The concentration of oxCCO measured with the UCLn algorithm increased during hyperoxia and correlated with brain tissue oxygen tension as measured by microdialysis; ${ }^{7}$ there was a negative correlation between oxCCO and lactate/ pyruvate ratio which shows that the oxCCO NIRS changes are related to cellular metabolism. Another investigation of six patients with TBI showed a mean increase in oxCCO during hypercapnia, but not in all individuals (four increase, two decrease) despite increases in Vmca-this could be due to heterogeneity of TBI. ${ }^{132}$ This study did not find an association between oxCCO and the lactate/pyruvate ratio-the authors suggest that this may be due to more complex changes induced by $\mathrm{CO}_{2}$, such as $\mathrm{pH}$, nitric oxide (NO) and cerebral metabolic rate of oxygen $\left(\mathrm{CMRO}_{2}\right)$ changes. ${ }^{132}$

Bed tilts were performed in patients with primary autonomic failure to assess cerebral oxCCO during hypotension measured with the NIRO $300.9^{93}$ There was a range of NIRS-measured oxCCO responses during large decrease in $\mathrm{HbD}$ and $\mathrm{HbT}$. In patients where a significant decrease in oxCCO was observed, there was a threshold in $\mathrm{HbD}$ and $\mathrm{HbT}$ reduction before it occurred. A similar threshold has been seen in piglet studies of anoxia, ${ }^{104}$ and may indicate the point at which damage to the cells due to hypoxia and/or ischemia occurs.

Another population at risk of hypoxic injury is OSA patients and these have been studied with the NIRO 300. The first study looked at OSA patients during daytime naps with NIRS monitoring, polysomnography, and laser Doppler. ${ }^{90}$ Correlations were seen during spontaneous hypoxias between changes in oxCCO and changes in TOI, $\mathrm{CBF}, \mathrm{SpO}_{2}$, and $\mathrm{MABP}$, there was a weak negative correlation between oxCCO and endtidal $\mathrm{CO}_{2}$. The oxCCO signal was in phase with TOI but not $\mathrm{HbO}_{2}$ or $\mathrm{HHb}$. These results suggest that OSA hypoxias cause anaerobic metabolism or $\mathrm{CO}_{2}$ changes to reduce $\mathrm{CCO}$. In another study of 62 sleep apnea patients, six had deep apneas (TOI drop of $>10 \%$ ) and oxCCO dropped for each apnea. ${ }^{133}$

\section{Discussion}

NIRS-measured oxCCO has been studied in many clinical environments. For neonates, this has included term and preterm 
infants, infants studied on neonatal intensive care units, and during cardiac surgery. The results from the neonatal cohort are not consistent, but that is largely due to the heterogeneity of the populations studied and differences between the protocols. An excellent example of the application of oxCCO in neonates is the indomethacin work, which has shown that the oxCCO signal can provide clinically important information..$^{70,73,77}$ The most recent work on neonatal HIE has shown that the relationship between cerebral oxCCO and oxygenation is indicative of the level of brain injury, as measured by a ${ }^{31} \mathrm{P}$ MRS biomarker of outcome, ${ }^{96}$ so oxCCO has potential clinical importance as a bedside marker of cerebral well-being.

The literature on adults also covers a wide range of clinical environments. The large study by Kakihana et al. ${ }^{130}$ is an excellent example of the potential of oxCCO to be a clinical tool that is predictive of neurological outcome after surgery. Further, there is a great potential for the oxCCO measurement to be used in fNIRS studies for a deeper understanding of oxygen metabolism during neurovascular coupling and for its brain specificity. $^{12}$

The work on adults also covers a portfolio of volunteer studies, which have been used to deepen our knowledge of the oxCCO signal in humans. The behavior of the oxidation state of CCO is not fully understood as there is no gold standard for the measurement in vivo. However, from the summary of the results found in this review, we are able to summarize the changes in its behavior from challenges performed on healthy adult volunteers. We are limited to the adult brain as it is not possible to obtain such controlled data on neonates. Quantification of a normal oxCCO response in a healthy brain will allow the identification of abnormal, and perhaps pathophysiological, cerebral responses. The generalized trends of the three NIRS chromophores in response to systemic changes are summarized in Fig. 8.

Interpretation of a change in the oxidation state of $\mathrm{CCO}$ is complex because it represents a change in equilibrium, which may reflect fluctuations in any of several factors and the balance between them including $\mathrm{O}_{2}$ supply, glucose or other substrate supply, $\mathrm{pH}$, temperature, and other ETC rates. Understanding the CCO signal is sometimes challenging as there are many different factors that can cause increased oxidation or reduction of the enzyme. This complexity can be dealt with by using computer modeling of the physiology. ${ }^{81}$

The UCL group has developed a mathematical model of the physiology of brain metabolism and circulation to aid the interpretation of the measurable cerebral signals. ${ }^{38-40,134}$ The first version of the model was an elaborate representation of the adult brain ${ }^{134}$ and was extended to simulate changes in the NIRS variables, in particular, changes in oxCCO. ${ }^{38}$ The model has been used with NIRS-based adult studies, ${ }^{38}$ and after modification for the piglet brain, ${ }^{39}$ has been used in controlled preclinical studies of HIE. ${ }^{40}$

\section{Future Directions}

Measuring a real-time, bedside marker of brain tissue metabolism is an active area of NIRS research. Many biomedical optics groups are measuring cerebral $\mathrm{CMRO}_{2}$ with combined NIRS and diffuse correlation spectroscopy. ${ }^{135-138}$ Others are combining MRI and NIRS to measure $\mathrm{CMRO}_{2},{ }^{139}$ confirming the need for a noninvasive measurement of tissue metabolism. We see NIRSmeasured oxCCO to be an alternative and/or complimentary measurement to these other monitors of cerebral metabolism.

NIR imaging or diffuse optical tomography is under continuous development. ${ }^{4}$ Recent work by Phan et al. ${ }^{105}$ demonstrates the first oxCCO images acquired using a multichannel broadband spectroscopy system during activation of the visual cortex (Fig. 9). This work has demonstrated differences between the localization of changes in oxCCO and the hemodynamic signals $\left(\mathrm{HbO}_{2}\right.$ and $\left.\mathrm{HHb}\right)$. This difference could be due to the oxCCO signal originating directly from the brain tissue, while the hemoglobin signals result from changes in the surrounding vasculature. Topographical images with oxCCO in addition to $\mathrm{HbO}_{2}$ and $\mathrm{HHb}$ will allow investigation of regional changes in cerebral oxygenation and oxygen consumption in the healthy and injured brain.

The most challenging but exciting foreseeable development for $\mathrm{CCO}$ would be an absolute measurement. To resolve changes in the oxidized concentration with respect to the reduced concentration of CCO would be revolutionary, as it would allow easier comparison between patients or volunteers, and give a better understanding of the state of tissue metabolism. The UCL group is working toward methods to do this, with both new hardware and algorithms. The group has recently developed a multiwavelength TR spectrometer to quantify absolute changes in absorption and scattering with a view to measuring absolute CCO redox changes. ${ }^{140,141}$

In terms of the future of the instrumentation, we believe that the capacity to measure changes in light attenuation using many wavelengths is a key in separating the contribution of CCO from the total attenuation spectra. While our recent theoretical work suggests that measurements at a specific combination of eight wavelengths (between 780 and $900 \mathrm{~nm}$ ) can accurately resolve oxCCO changes to $2 \%$ difference when compared to a broadband (121 wavelengths) measurement, ${ }^{62}$ we have yet to develop and test an instrument that implements this solution. Instruments based on broadband spectrometers with the capacity to measure the changes in light attenuation at more than 100 wavelengths have been extremely successful in separating the CCO signal from the hemoglobins and are considered a key component to an optimal CCO instrument. Broadband spectrometer

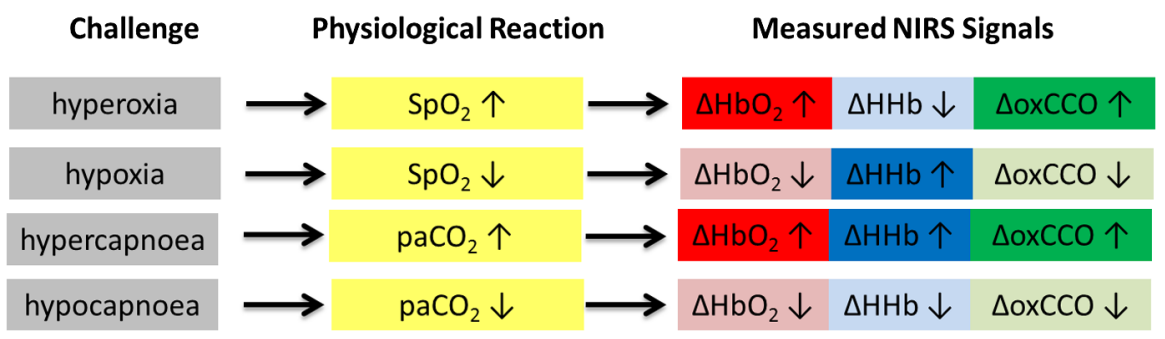

Fig. 8 Physiological stimuli and predicted NIRS-measured hemoglobin oxygenation and oxCCO changes based on findings from this review for the healthy adult brain. 


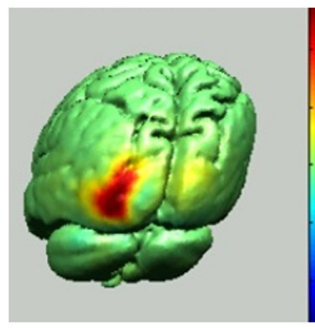

$\mathrm{HbO}_{2}$

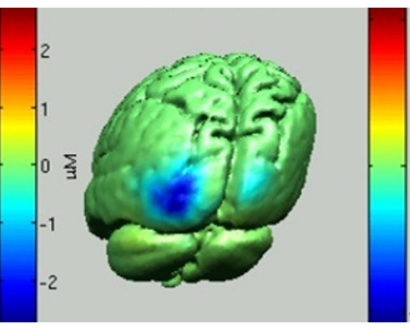

$\mathrm{HHb}$

(b)

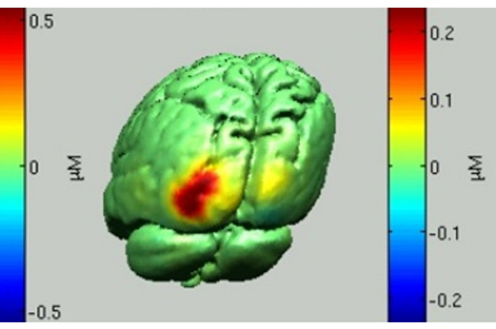

$\mathrm{OxCCO}$

(c)

Fig. 9 Images of block-averaged concentration changes over the occipital lobe from (a) $\mathrm{HbO}_{2}$, (b) $\mathrm{HHb}$, and (c) oxCCO for one subject. Images are recorded during half-field checkerboard visual stimulation, demonstrating spatial distribution of responses. There is an increase in $\mathrm{HbO}_{2}$ and oxCCO on the contralateral hemisphere during activation and a decrease in $\mathrm{HHb}$. There are spatial differences between the hemoglobin and oxCCO images as assessed by center of gravity analysis. This figure has been produced from data presented by Phan et al. ${ }^{105}$

instrumentation has advanced significantly the last 30 years and we now have systems with enhanced light throughput and photon sensitivity in the NIR, with reduced noise and improved electronics for fast acquisition, so they are robust enough to be used in a clinical setting. ${ }^{96}$ In addition, through advances in laser instrumentation and the development of supercontinuum fiber laser sources, we now have the capacity to perform timeof-flight measurements of multiple wavelengths of our choice, allowing us to resolve tissue light absorption and scattering across the NIR spectra to quantify oxCCO. ${ }^{140-142}$ Ten years from now, we see NIRS instruments and cerebral oximeters enhanced with the added capacity to measure oxCCO through the utilization of an increased number of wavelengths.

\section{Conclusion}

In this review, we described the rationale, methods and analysis behind NIRS measures of oxCCO. We summarized the range of human brain studies (in healthy volunteers and patients) from those first performed by the Jöbsis group, as early as 1985, to the present day. We found 103 papers published on cerebral oxCCO NIRS measurements in humans, $65 \%$ of the studies are on adults, and the remaining $35 \%$ on neonates. The need for a low-cost, bedside and noninvasive monitor of cerebral oxygen metabolism remains an important and relevant goal for both clinical and functional brain studies. This unmet need has driven continued advances to deliver NIRS instrumentation and methodologies, which are optimized specifically for CCO measures. With this comes renewed focus on defining the role of the $\mathrm{CCO}$ NIRS measurements in a range of clinical scenarios and fulfilling Jöbsis's vision for this optically derived metabolic marker.

\section{References}

1. F. Jöbsis, "Noninvasive, infrared monitoring of cerebral and myocardial oxygen sufficiency and circulatory parameters," Science 198(4323), 1264-1267 (1977).

2. F. Jöbsis-Vandervliet, "Discovery of the near-infrared window into the body and the early development of near-infrared spectroscopy," J. Biomed. Opt. 4(4), 392-396 (1999).

3. M. Wolf, M. Ferrari, and V. Quaresima, "Progress of near-infrared spectroscopy and topography for brain and muscle clinical applications," J. Biomed. Opt. 12(6), 062104 (2007).

4. F. Scholkmann et al., "A review on continuous wave functional nearinfrared spectroscopy and imaging instrumentation and methodology," Neuroimage 85(Pt 1), 6-27 (2014).
5. S. Suzuki et al., "A tissue oxygenation monitor using NIR spatially resolved spectroscopy," Proc. SPIE 3597, 582-592 (1999).

6. S. Hyttel-Sorensen et al., "Cerebral near infrared spectroscopy oximetry in extremely preterm infants: phase II randomised clinical trial," Br. Med. J. 350, g7635 (2015).

7. M. Tisdall et al., "Increase in cerebral aerobic metabolism by normobaric hyperoxia after traumatic brain injury," J. Neurosurg. 109(3), 424-432 (2008).

8. T. Shin'oka et al., "Utility of near-infrared spectroscopic measurements during deep hypothermic circulatory arrest," Ann. Thorac. Surg. 69(2), 578-583 (2000).

9. C. Peeters-Scholte et al., "Redox state of near infrared spectroscopymeasured cytochrome $\mathrm{aa}_{3}$ correlates with delayed cerebral energy failure following perinatal hypoxia-ischaemia in the newborn pig," Exp. Brain Res. 156(1), 20-26 (2004).

10. A. Bainbridge et al., "Brain mitochondrial oxidative metabolism during and after cerebral hypoxia-ischemia studied by simultaneous phosphorus magnetic-resonance and broadband near-infrared spectroscopy," Neuroimage 102(Pt. 1), 173-83 (2013).

11. M. Tsuji et al., "Reduction of cytochrome aa3 measured by near-infrared spectroscopy predicts cerebral energy loss in hypoxic piglets," Pediatr. Res. 37(3), 253-259 (1995).

12. C. Kolyva et al., "Systematic investigation of changes in oxidized cerebral cytochrome $\mathrm{c}$ oxidase concentration during frontal lobe activation in healthy adults," Biomed. Opt. Express 3(10), 2550-2566 (2012).

13. C. Kolyva et al., "Cytochrome c oxidase response to changes in cerebral oxygen delivery in the adult brain shows higher brain-specificity than haemoglobin," Neuroimage 85(Pt. 1), 234-244 (2014).

14. I. Tachtsidis and F. Scholkmann, "False positives and false negatives in functional NIRS: 7 issues, challenges and the way forward," Neurophotonics 3(3), 031405 (2016).

15. H. Matsumoto et al., "Does the redox state of cytochrome aa3 reflect brain energy level during hypoxia? Simultaneous measurements by near infrared spectrophotometry and ${ }^{31} \mathrm{P}$ nuclear magnetic resonance spectroscopy," Anesth. Analg. 83(3), 513-518 (1996).

16. C. E. Cooper et al., "Use of mitochondrial inhibitors to demonstrate that cytochrome oxidase near-infrared spectroscopy can measure mitochondrial dysfunction noninvasively in the brain," J. Cereb. Blood Flow Metab. 19(1), 27-38 (1999).

17. V. Quaresima et al., "Oxidation and reduction of cytochrome oxidase in the neonatal brain observed by in vivo near-infrared spectroscopy," Biochim. Biophys. Acta-Bioenerg. 1366(3), 291-300 (1998).

18. M. Ferrari et al., "Non-invasive near infrared spectroscopy of brain in fluorocarbon exchange-transfused rats," Physiol. Chem. Phys. Med. NMR 15(2), 107-113 (1983).

19. Y. Hoshi et al., "Redox behavior of cytochrome oxidase in the rat brain measured by near-infrared spectroscopy," J. Appl. Physiol. 83(6), 18421848 (1997).

20. Y. Hoshi and M. Tamura, "Dynamic changes in cerebral oxygenation in chemically induced seizures in rats: study by near-infrared spectrophotometry," Brain Res. 603(2), 215-221 (1993). 
21. C. A Piantadosi and F. Jöbsis-Vandervliet, "Spectrophotometry of cerebral cytochrome a, a3 in bloodless rats," Brain Res. 305(1), 89-94 (1984).

22. J. Brazy et al., "Noninvasive monitoring of cerebral oxygenation in preterm infants: preliminary observations," Pediatrics 75(2), 217-225 (1985).

23. C. E. Cooper and R. Springett, "Measurement of cytochrome oxidase and mitochondrial energetics by near-infrared spectroscopy," Philos. Trans. R. Soc. Lond. B. Biol. Sci. 352(1354), 669-676 (1997).

24. K. Uludağ et al., "Cytochrome-c-oxidase redox changes during visual stimulation measured by near-infrared spectroscopy cannot be explained by a mere cross talk artefact," Neuroimage 22(1), 109-119 (2004).

25. I. Tachtsidis et al., "A hybrid multi-distance phase and broadband spatially resolved spectrometer and algorithm for resolving absolute concentrations of chromophores in the near-infrared light spectrum," Adv. Exp. Med. Biol. 662, 169-175 (2010).

26. J. C. LaManna, "The redox state of cytochrome oxidase in brain in vivo: an historical perspective," Adv. Exp. Med. Biol. 530, 535-546 (2003).

27. W. G. Zijlstra, A. Buursma, and W. P. Meeuwsen-van der Roest, "Absorption spectra of human fetal and adult oxyhemoglobin, de-oxyhemoglobin, carboxyhemoglobin, and methemoglobin," Clin. Chem. 37(9), 1633-1638 (1991).

28. M. Cope, "The application of near infrared spectroscopy to non invasive monitoring of cerebral oxygenation in the newborn infant," PhD Thesis, University College London (1991).

29. Biomedical Optics Research Laboratory, UCL Department of Medical Physics and Biomedical Engineering "Tissue spectra," 2005, http:// www.ucl.ac.uk/medphys/research/borl/intro/spectra (22 April 2016).

30. M. G. Mason, P. Nicholls, and C. E. Cooper, "Re-evaluation of the near infrared spectra of mitochondrial cytochrome c oxidase: implications for non invasive in vivo monitoring of tissues," Biochim. Biophys. Acta 1837(11), 1882-1891 (2014).

31. C. E. Cooper et al., "Near-infrared spectroscopy of the brain: relevance to cytochrome oxidase bioenergetics," Biochem. Soc. Trans. 22(4), 974980 (1994).

32. M. Banaji, "A generic model of electron transport in mitochondria," J. Theor. Biol. 243(4), 501-516 (2006).

33. H. R. Heekeren et al., "Noninvasive assessment of changes in cytochrome-c oxidase oxidation in human subjects during visual stimulation," J. Cereb. Blood Flow Metab. 19(6), 592-603 (1999).

34. J. P. Newman et al., "Hemodynamic and metabolic responses to moderate asphyxia in brain and skeletal muscle of late-gestation fetal sheep," J. Appl. Physiol. 88(1), 82-90 (2000).

35. I. Tachtsidis et al., "Relationship between brain tissue haemodynamics, oxygenation and metabolism in the healthy human adult brain during hyperoxia and hypercapnea," Adv. Exp. Med. Biol. 645, 315-320 (2009).

36. G. C. Brown, M. Crompton, and S. Wray, "Cytochrome oxidase content of rat brain during development," Biochim. Biophys. Acta 1057(2), 273275 (1991).

37. C. Cooper, D. Delpy, and E. Nemoto, "The relationship of oxygen delivery to absolute haemoglobin oxygenation and mitochondrial cytochrome oxidase redox state in the adult brain: a near-infrared spectroscopy study," Biochem. J. 332(Pt. 3), 627-632 (1998).

38. M. Banaji et al., "A model of brain circulation and metabolism: NIRS signal changes during physiological challenges," PLoS Comput. Biol. 4(11), e1000212 (2008).

39. T. Moroz et al., "Computational modelling of the piglet brain to simulate near-infrared spectroscopy and magnetic resonance spectroscopy data collected during oxygen deprivation," J. R. Soc. Interface 9(72), 1499-1509 (2012).

40. M. Caldwell et al., "Brainsignals revisited: simplifying a computational model of cerebral physiology," PLoS One 10(5), e0126695 (2015).

41. S. J. Matcher et al., "Performance comparison of several published tissue near-infrared spectroscopy algorithms," Anal. Biochem. 227(1), 54-68 (1995).

42. D. Delpy and M. Cope, "Estimation of optical pathlength through tissue from direct time of flight measurement," Phys. Med. Biol. 33(12), 14331442 (1988).

43. M. Cope et al., "Data analysis methods for near-infrared spectroscopy of tissue: problems in determining the relative cytochrome aa3 concentration," Proc. SPIE 1431, 251-263 (1991).
44. Y. Wickramasinghe et al., "Effect of fetal hemoglobin on the determination of neonatal cerebral oxygenation by near-infrared spectroscopy," Pediatr. Res. 34(1), 15-17 (1993).

45. O. Hazeki and M. Tamura, "Quantitative analysis of hemoglobin oxygenation state of rat brain in situ by near-infrared spectrophotometry," J. Appl. Physiol. 64(2), 796-802 (1988).

46. Y. Kakihana et al., "Brain oxymetry in the operating room: current status and future directions with particular regard to cytochrome oxidase," J. Biomed. Opt. 13(3), 033001 (2008).

47. C. Piantadosi, "Absorption spectroscopy for assessment of mitochondrial function in vivo," Methods Toxicol. 2, 107-126 (1993).

48. H. Miyake et al., "The detection of cytochrome oxidase heme iron and copper absorption in the blood-perfused and blood-free brain in normoxia and hypoxia," Anal. Biochem. 192(1), 149-155 (1991).

49. S. Wray et al., "Characterization of the near infrared absorption spectra of cytochrome aa3 and haemoglobin for the non-invasive monitoring of cerebral oxygenation," Biochim. Biophys. Acta 933(1), 184-192 (1988).

50. A. Matsunaga et al., "Energy-dependent redox state of heme a + a3 and copper of cytochrome oxidase in perfused rat brain in situ," Am. J. Physiol. 275(4 Pt 1), 1022-1030 (1998).

51. R. Stingele et al., "Reduction of cytochrome-c oxidase copper precedes failing cerebral $\mathrm{O}_{2}$ utilization in fluorocarbon-perfused cats," Am. J. Physiol. 271(2), H579-H587 (1996).

52. M. Ferrari et al., "Redox changes in cat brain cytochrome-c oxidase after blood-fluorocarbon exchange," Am. J. Physiol. 258(6 Pt 2), 1706-1713 (1990).

53. M. Ferrari et al., "Cat brain cytochrome-c oxidase redox changes induced by hypoxia after blood-fluorocarbon exchange transfusion," Am. J. Physiol. 269(2 Pt 2), H417-H424 (1995).

54. S. R. Arridge, M. Cope, and D. T. Delpy, "The theoretical basis for the determination of optical pathlengths in tissue: temporal and frequency analysis," Phys. Med. Biol. 37(7), 1531-1560 (1992).

55. A Duncan et al., "Optical pathlength measurements on adult head, calf and forearm and the head of the newborn infant using phase resolved optical spectroscopy," Phys. Med. Biol. 40(2), 295-304 (1995).

56. S. J. Matcher, M. Cope, and D. T. Delpy, "Use of the water absorption spectrum to quantify tissue chromophore concentration changes in nearinfrared spectroscopy," Phys. Med. Biol. 39(1), 177-196 (1994).

57. M. Essenpreis et al., "Spectral dependence of temporal point spread functions in human tissues," Appl. Opt. 32(4), 418-425 (1993).

58. M. Tisdall et al., "Changes in the attenuation of near infrared spectra by the healthy adult brain during hypoxaemia cannot be accounted for solely by changes in the concentrations of oxy- and deoxy-haemoglobin," Adv. Exp. Med. Biol. 614, 217-225 (2008).

59. A. Ghosh et al., "Normobaric hyperoxia does not change optical scattering or pathlength but does increase oxidised cytochrome c oxidase concentration in patients with brain injury," $A d v$. Exp. Med. Biol. 765, 67-72 (2013).

60. L. Skov and G. Greisen, "Apparent cerebral cytochrome aa 3 reduction during cardiopulmonary bypass in hypoxaemic children with congenital heart disease. A critical analysis of in vivo near-infrared spectrophotometric data," Physiol. Meas. 15(4), 447 (1994).

61. J. S. Maier et al., "Possible correlation between blood glucose concentration and the reduced scattering coefficient of tissues in the near infrared," Opt. Lett. 19(24), 2062-2064 (1994).

62. D. Arifler et al., "Optimal wavelength combinations for near-infrared spectroscopic monitoring of changes in brain tissue hemoglobin and cytochrome c oxidase concentrations," Biomed. Opt. Express 6(3), 933 (2015).

63. J. E. Brazy and D. V Lewis, "Changes in cerebral blood volume and cytochrome aa3 during hypertensive peaks in preterm infants," J. Pediatr. 108(6), 983-987 (1986).

64. J. E. Brazy, "Effects of crying on cerebral blood volume and cytochrome aa3," J. Pediatr. 112(3), 457-461 (1988).

65. J. S. Wyatt et al., "Quantification of cerebral oxygenation and haemodynamics in sick newborn infants by near infrared spectrophotometry," Lancet 328(8515), 1063-1066 (1986).

66. W. J. Greeley et al., "The effect of hypothermic cardiopulmonary bypass and total circulatory arrest on cerebral metabolism in neonates, infants, and children," J. Thorac. Cardiovasc. Surg. 101(5), 783-794 (1991). 
67. D. S. Bacon et al., "Cerebrocortical oxygenation and ventilatory response during sustained hypoxia," Respir. Physiol. 80(2-3), 245257 (1990).

68. N. B. Hampson et al., "Cerebral oxygen availability by NIR spectroscopy during transient hypoxia in humans," J. Appl. Physiol. 69(3), 907-913 (1990).

69. F. van Bel et al., "Changes in cerebral hemodynamics and oxygenation in the first 24 hours after birth asphyxia," Pediatrics 92(3), 365-372 (1993).

70. M. J. Benders et al., "Acute effects of indomethacin on cerebral hemodynamics and oxygenation," Biol. Neonate 68(2), 91-99 (1995).

71. O. Pryds et al., "Carbon dioxide-related changes in cerebral blood volume and cerebral blood flow in mechanically ventilated preterm neonates: comparison of near infrared spectrophotometry and 133 Xenon clearance," Pediatr. Res. 27(5), 445-449 (1990).

72. A. Edwards, J. Wyatt, and C. Richardson, "Effects of indomethacin on cerebral haemodynamics in very preterm infants," Lancet 335(8704), 1491-1495 (1990).

73. D. C. McCormick et al., "Effect of indomethacin on cerebral oxidized cytochrome oxidase in preterm infants," Pediatr. Res. 33(6), 603-608 (1993).

74. H. U. Bucher et al., "Effect of aminophylline on cerebral haemodynamics and oxidative metabolism in premature infants," Eur. J. Pediatr. 153(2), 123-128 (1994).

75. P. Casaer et al., "Cytochrome aa 3 and intracranial pressure in newborn infants; a near infrared spectroscopy study," Neuropediatrics 23(2), 111 (1992).

76. K. D. Liem et al., "The effect of blood transfusion and haemodilution on cerebral oxygenation and haemodynamics in newborn infants investigated by near infrared spectrophotometry," Eur. J. Pediatr. 156(4), 305-310 (1997).

77. K. D. Liem et al., "Effects of repeated indomethacin administration on cerebral oxygenation and haemodynamics in preterm infants: combined near infrared spectrophotometry and Doppler ultrasound study," Eur. J. Pediatr. 153(7), 504-509 (1994).

78. G. Grubhofer et al., "A comparison of the monitors INVOS 3100 and NIRO 500 in detecting changes in cerebral oxygenation," Acta Anaesthesiol. Scand. 43(4), 470-475 (1999).

79. P. D. Adelson et al., "Noninvasive continuous monitoring of cerebral oxygenation periictally using near-infrared spectroscopy: a preliminary report," Epilepsia 40(11), 1484-1489 (1999).

80. B. Urlesberger et al., "Changes in cerebral blood volume and cerebral oxygenation during periodic breathing in term infants," Neuropediatrics 31(2), 75-81 (2000).

81. J. G. LeBlanc et al., "Effects of propofol on cerebral oxygenation during cardiopulmonary bypass in children," Can. J. Anaesth. 47(11), 10821089 (2000).

82. C. Kohlhauser et al., "Effects of endotracheal suctioning in highfrequency oscillatory and conventionally ventilated low birth weight neonates on cerebral hemodynamics observed by near infrared spectroscopy (NIRS)," Pediatr. Pulmonol. 29(4), 270-275 (2000).

83. J. S. Soul et al., "CSF removal in infantile posthemorrhagic hydrocephalus results in significant improvement in cerebral hemodynamics," Pediatr. Res. 55(5), 872-876 (2004).

84. G. Nollert et al., "Oxygenation strategy and neurologic damage after deep hypothermic circulatory arrest. I. Gaseous microemboli," $J$. Thorac. Cardiovasc. Surg. 117(6), 1166-1171 (1999).

85. A. J. Du Plessis et al., "Cerebral oxygen supply and utilization during infant cardiac surgery," Ann. Neurol. 37(4), 488-497 (1995).

86. P. Zaramella et al., "Brain auditory activation measured by nearinfrared spectroscopy (NIRS) in neonates," Pediatr. Res. 49(2), 213219 (2001).

87. A. Azakie et al., "Cerebral oxygen balance is impaired during repair of aortic coarctation in infants and children," J. Thorac. Cardiovasc. Surg. 130(3), 830-836 (2005).

88. N. Nagdyman et al., "Cerebral oxygenation measured by near-infrared spectroscopy during circulatory arrest and cardiopulmonary resuscitation," Br. J. Anaesth. 91(3), 438-442 (2003).

89. C. Dani et al., "Brain hemodynamic changes in preterm infants after maintenance dose caffeine and aminophylline treatment," Biol. Neonate 78(1), 27-32 (2000).
90. A. McGown et al., "Measurement of changes in cytochrome oxidase redox state during obstructive sleep apnea using near-infrared spectroscopy," Sleep 26(6), 710-716 (2003).

91. E. McNeill et al., "Cerebral oxygenation during defibrillator threshold testing of implantable cardioverter defibrillators," Pacing Clin. Electrophysiol. 28(6), 528-533 (2005).

92. I. Tachtsidis et al., "Changes in cerebral total haemoglobin volume and cytochrome oxidase redox state during deep apnoeas in patients with obstructive sleep apnoea," in Biomedical Topical Meeting, OSA Technical Digest, paper WF6 (2004).

93. I. Tachtsidis et al., "Investigation of in vivo measurement of cerebral cytochrome-c-oxidase redox changes using near-infrared spectroscopy in patients with orthostatic hypotension," Physiol. Meas. 28(2), 199211 (2007).

94. M. Wolf et al., "Do slow and small oxygen changes affect the cerebral cytochrome oxidase redox state measurement by near-infrared spectroscopy?," Adv. Exp. Med. Biol. 578, 245-250 (2006).

95. C. E. Cooper et al., "Measurement of cytochrome oxidase redox state by near infrared spectroscopy," Adv. Exp. Med. Biol. 413, 63-73 (1997).

96. G. Bale et al., "A new broadband near-infrared spectroscopy system for in-vivo measurements of cerebral cytochrome-c-oxidase changes in neonatal brain injury," Biomed. Opt. Express 5(10), 3450-3466 (2014).

97. H. Obrig et al., "Spontaneous low frequency oscillations of cerebral hemodynamics and metabolism in human adults," Neuroimage 12(6), $623-639$ (2000).

98. K. Uludag et al., "Cross talk in the Lambert-Beer calculation for near-infrared wavelengths estimated by Monte Carlo simulations," J. Biomed. Opt. 7(1), 51-59 (2002).

99. P. Wobst et al., "Linear aspects of changes in deoxygenated hemoglobin concentration and cytochrome oxidase oxidation during brain activation," Neuroimage 13(3), 520-530 (2001).

100. A. Ghosh et al., "Use of a hybrid optical spectrometer for the measurement of changes in oxidized cytochrome c oxidase concentration and tissue scattering during functional activation," Adv. Exp. Med. Biol. 737, 119-124 (2012).

101. I. Tachtsidis et al., "Multi-wavelength, depth resolved, scattering and pathlength corrected in vivo near-infrared spectroscopy of brain tissue," in Biomedical Optics and 3-D Imaging, OSA Technical Digest, paper BTuB7 (2010).

102. M. Mitchell, An Introduction to Genetic Algorithms (Complex Adaptive Systems), MIT Press, Massachusetts, (1998).

103. T. Zhu et al., "Optimal wavelength combinations for resolving in-vivo changes of haemoglobin and cytochrome-c-oxidase concentrations with NIRS," in Biomedical Optics and 3-D Imaging, OSA Technical Digest, paper JM3A.6 (2012).

104. R. Springett et al., "Oxygen dependency and precision of cytochrome oxidase signal from full spectral NIRS of the piglet brain," Am. $J$. Physiol. Heart Circ. Physiol. 279(5), H2202-H2209 (2000).

105. P. Phan et al., "Spatial distribution of changes in oxidised cytochrome c oxidase during visual stimulation using broadband near infrared spectroscopy imaging," Adv Exp Med Biol. (in press).

106. M. Diop et al., "Improved light collection and wavelet de-noising enable quantification of cerebral blood flow and oxygen metabolism by a low-cost, off-the-shelf spectrometer," J. Biomed. Opt. 19(5), 057007 (2014).

107. T. Muehlemann, D. Haensse, and M. Wolf, "Wireless miniaturized in-vivo near infrared imaging," Opt. Express 16(14), 10323-10330 (2008).

108. D. Chitnis et al., "Towards a wearable near infrared spectroscopic probe for monitoring concentrations of multiple chromophores in biological tissue in vivo," (under review).

109. F. Bevilacqua et al., "Broadband absorption spectroscopy in turbid media by combined frequency-domain and steady-state methods," Appl. Opt. 39(34), 6498-6507 (2000).

110. E. Okada and D. T. Delpy, "Near-infrared light propagation in an adult head model I modeling of low-level scattering in the cerebrospinal fluid layer," Appl. Opt. 42(16), 2906 (2003).

111. E. Okada and D. T. Delpy, "Near-infrared light propagation in an adult head model. II. effect of superficial tissue thickness on the sensitivity of the near-infrared spectroscopy signal," Appl. Opt. 42(16), 2915 (2003). 
112. Y. Fukui, Y. Ajichi, and E. Okada, "Monte Carlo prediction of nearinfrared light propagation in realistic adult and neonatal head models," Appl. Opt. 42(16), 2881 (2003).

113. S. Brigadoi and R. J. Cooper, "How short is short? Optimum source detector distance for short-separation channels in functional near-infrared spectroscopy," Neurophotonics 2(2) (2015).

114. M. Dehaes et al., "Assessment of the frequency domain multi-distance method to evaluate the brain optical properties: Monte Carlo simulations from neonate to adult," Biomed. Opt. Express 2(3), 552-567 (2011).

115. K. Blomgren et al., "Free radicals, mitochondria, and hypoxia-ischemia in the developing brain," Free Radical Biol. Med. 40(3), 388-397 (2006).

116. M. Tisdall et al., "Near-infrared spectroscopic quantification of changes in the concentration of oxidized cytochrome $\mathrm{c}$ oxidase in the healthy human brain during hypoxemia," J. Biomed. Opt. 12(2), 024002 (2007).

117. A. D. Edwards et al., "Quantification of concentration changes in neonatal human cerebral oxidized cytochrome oxidase," J. Appl. Physiol. 71(5), 1907-1913 (1991).

118. Y. A. B. D. Wickramasinghe et al., "Investigation of neonatal brain cytochrome redox by NIRS," Brain Res. Dev. Brain Res. 89(2), 307-308 (1995).

119. N. Nagdyman et al., "Comparison between cerebral tissue oxygenation index measured by near-infrared spectroscopy and venous jugular bulb saturation in children," Intensive Care Med. 31(6), 846-850 (2005).

120. K. C. Sekar and K. E. Corff, "Treatment of patent ductus arteriosus: indomethacin or ibuprofen?," J. Perinatol. 28(Suppl 1), S60-S62 (2008).

121. J. S. Wyatt et al., "Magnetic resonance and near infrared spectroscopy for investigation of perinatal hypoxic-ischaemic brain injury," Arch. Dis. Child. 64(7), 953-963 (1989).

122. S. Mitra et al., "Relationship between cerebral oxygenation and metabolism during rewarming in newborn infants after therapeutic hypothermia following hypoxic-ischemic brain injury," $A d v$. Exp. Med. Biol (in press).

123. G. Bale et al., "Interrelationship between broadband NIRS measurements of cerebral cytochrome-c-oxidase and systemic changes indicates injury severity in neonatal encephalopathy," Adv. Exp. Med. Biol (in press).

124. S. Mitra et al., "In vivo measurement of cerebral mitochondrial metabolism using broadband near infrared spectroscopy following neonatal stroke," Adv. Exp. Med. Biol 876, 493-499 (2016).

125. A. Villringer et al., "Near infrared spectroscopy (NIRS): a new tool to study hemodynamic changes during activation of brain function in human adults," Neurosci. Lett. 154(1-2), 101-104 (1993).

126. M. Tisdall et al., "Changes in concentrations of oxidised cytochrome oxidase measured using both broadband and four wavelength near infrared spectroscopy reflect changes in oxygen delivery during hypoxaemia in healthy volunteers," in Biomedical Optics, OSA Technical Digest, paper ME66 (2006).

127. A. Ghosh et al., "Reduction of cytochrome c oxidase during vasovagal hypoxia-ischaemia in human adult brain: a case study," Adv. Exp. Med. Biol. 789, 21-27 (2012).

128. I. de Roever et al., "Broadband NIRS measured cytochrome-c-oxidase shows higher brain-specificity than haemoglobin in functional activation," in Biomedical Optics, OSA Technical Digest (2016).

129. G. Nollert, T. Shin'oka, and R. A. Jonas, "Near-infrared spectrophotometry of the brain in cardiovascular surgery," Thorac. Cardiovasc. Surg. 46(3), 167-175 (1998).

130. Y. Kakihana et al., "Redox behavior of cytochrome oxidase and neurological prognosis in 66 patients who underwent thoracic aortic surgery," Eur. J. Cardiothorac. Surg. 21(3), 434-439 (2002).
131. M. Ferrari et al., "Effects of carotid compression, as assessed by near infrared spectroscopy, upon cerebral blood volume and haemoglobin oxygen saturation," J. R. Soc. Med. 80(2), 83-87 (1987).

132. I. Tachtsidis et al., "Analysis of the changes in the oxidation of brain tissue cytochrome-c-oxidase in traumatic brain injury patients during hypercapnoea," Adv. Exp. Med. Biol. 701, 9-14 (2011).

133. I. Tachtsidis et al., "Changes in cerebral total haemoglobin volume and cytochrome oxidase redox state during deep apnoeas in patients with obstructive sleep apnoea," in Biomedical Topical Meeting, OSA Technical Digest, paper WF6 (2004).

134. M. Banaji et al., "A physiological model of cerebral blood flow control," Math. Biosci. 194(2), 125-173 (2005).

135. N. Roche-Labarbe et al., "Noninvasive optical measures of CBV, $\mathrm{StO} 2$, CBF index, and rCMRO2 in human premature neonates' brains in the first six weeks of life," Hum. Brain Mapp. 31(3), 341-352 (2010).

136. K. M. Tichauer et al., "Using near-infrared spectroscopy to measure cerebral metabolic rate of oxygen under multiple levels of arterial oxygenation in piglets," J. Appl. Physiol. 109(3), 878-885 (2010).

137. N. Roche-Labarbe et al., "Near-infrared spectroscopy assessment of cerebral oxygen metabolism in the developing premature brain," J. Cereb. Blood Flow Metab. 32(3), 481-488 (2012).

138. M. Dehaes et al., "Cerebral oxygen metabolism in neonatal hypoxic ischemic encephalopathy during and after therapeutic hypothermia," J. Cereb. Blood Flow Metab. 34(1), 87-94 (2014).

139. P. Wintermark et al., "Near-infrared spectroscopy versus magnetic resonance imaging to study brain perfusion in newborns with hypoxicischemic encephalopathy treated with hypothermia," Neuroimage 85(Pt 1), 287-293 (2014).

140. L. Dunne, J. Hebden, and I. Tachtsidis, "Development of a near infrared multi-wavelength, multi-channel, time-resolved spectrometer for measuring brain tissue haemodynamics and metabolism," Adv. Exp. Med. Biol. 812, 181-186 (2014).

141. L. Dunne, J. Hebden, and I. Tachtsidis, "Supercontinuum multiwavelength, multi-channel, time-resolved near infrared spectrometer," in Biomedical Optics, OSA Technical Digest, paper BS3A.43 (2014).

142. F. Lange, F. Peyrin, and B. Montcel, "A hyperspectral time resolved DOT system to monitor physiological changes of the human brain activity," Proc. SPIE 9536, 95360R (2015).

Gemma Bale is a research associate in the Biomedical Optics Research Laboratory in the Department of Medical Physics and Biomedical Engineering at UCL. Her work focuses developing new near-infrared spectroscopy (NIRS) techniques for the measurement of cerebral oxygenation and metabolism, via cytochrome-c-oxidase. In particular, her PhD involved developing and demonstrating a novel broadband NIRS instrument to monitor neonatal brain injury in the intensive care unit.

Clare E. Elwell is a professor of medical physics in the Department of Medical Physics and Biomedical Engineering at UCL. She leads the near infrared spectroscopy (NIRS) research group developing novel optical systems for monitoring and imaging the human body and brain. Her research projects include studies of autism, acute brain injury, sports performance, migraine, malaria, depression, and, most recently, the effects of malnutrition on brain development with the first infant functional brain imaging study in Africa.

Ilias Tachtsidis received his $\mathrm{PhD}$ at the University College London (UCL), UK, in 2005. He is a senior member of the Biomedical Optics Research Laboratory at UCL and a senior Wellcome Trust fellow. He works on the development and application of NIRS techniques to monitor the function of the brain both in health and disease, including traumatic brain injury, and neonatal encephalopathy. 